\title{
Review
}

\section{X-ray studies of neutron stars and their magnetic fields}

\author{
By Kazuo MAKISHIMA*1, $†$
}

(Communicated by Yasuo TANAKA, M.J.A.)

\begin{abstract}
Utilizing results obtained over the past quarter century mainly with Japanese $\mathrm{X}$-ray astronomy satellites, a review is given to some aspects of neutron stars (NSs), with a particular emphasis on the magnetic fields (MFs) of mass-accreting NSs and magnetars. Measurements of electron cyclotron resonance features in binary X-ray pulsars, using the Ginga and Suzaku observatories, clarified that their surface MFs are concentrated in a narrow range of $(1-7) \times 10^{8} \mathrm{~T}$. Extensive studies of magnetars with Suzaku reinforced their nature as neutron stars with truly strong MFs, and revealed several important clues to their formation, evolution, and physical states. Taking all these results into account, a discussion is made on the origin and evolution of these strong MFs. One possible scenario is that the MF of NSs is a manifestation of some fundamental physics, e.g., neutron spin alignment or chirality violation, and the MF makes transitions from strong to weak states.
\end{abstract}

Keywords: neutron stars, pulsars, magnetars, magnetic fields, X-rays, scientific satellites

\section{Introduction}

The present paper deals mainly with magnetic fields (MFs) of neutron stars (NSs). Let us, however, begin with a broader scope, and explain how the concept of stars is directly related to basic physics. This is because the paper is meant for readers from wide areas of physical science, rather than experts in astrophysics. For the same reason, we spare considerable pages to tutorial explanations of the basics of NSs, and use the International System Units.

Generally, a "star" means a celestial system, in which gravity is counter-balanced in a stable manner by some internal pressure. The gravity, which provides the inward pull force, is universal without any characteristic length scales. In contrast, the pressure $p$ which tries to expand the star is versatile. It is indeed the variety of this $p$ that allows the existence of stars of various types. More specifically, stars are characterized almost solely by the equation of state (EOS), which expresses $p$ in terms of the mass density $\rho$ of the constituent matter.

*1 MAXI Team, Global Research Cluster, The Institute of Physical and Chemical Research (RIKEN), Wako, Saitama, Japan.

$\dagger$ Correspondence should be addressed: K. Makishima, MAXI Team, Global Research Cluster, The Institute of Physical and Chemical Research (RIKEN), 2-1 Hirosawa, Wako, Saitama 351-0198, Japan (e-mail: maxima@riken.jp).
As illustrated in Fig. 1, the differences in the EOS are best distinguished by the relation between the mass $M$ and radius $R$ of the stars. Normal nuclear-burning stars are supported by the classical gas pressure of their interior. In contrast, planets are supported by Coulomb repulsion among constituent ions, which determines the mean particle separation $d$ to be about the Bohr radius $a_{\mathrm{B}}$, and produces a scaling as $R \propto M^{1 / 3}$ (i.e., a constant density).

In brown dwarfs (stars too light to ignite hydrogen fusion) and white dwarfs (final form of low-mass stars), the gravity is counter-balanced by degenerate election pressure. Thus, $d$ becomes comparable to the electron Compton wavelength, $\lambda_{\mathrm{e}}=$ $2 \pi \alpha_{\mathrm{E}} a_{\mathrm{B}}=0.046 a_{\mathrm{B}}$, where $\alpha_{\mathrm{E}}=e^{2} / 4 \pi \epsilon_{0} \hbar c=1 / 137$ is the fine structure constant, with $e$ the elementary charge, $\varepsilon_{0}$ the vacuum dielectric constant, $\hbar$ the Dirac constant, and $\mathrm{c}$ the light velocity. In non-relativistic case, the mass-radius scaling becomes $R \propto M^{-1 / 3}$. When the degenerate Fermions become fully relativistic, the star becomes unstable. This sets an upper limit to these objects. For white dwarfs, the limit, called Chandrasekhar mass, is given as $\sim m_{\mathrm{p}} \alpha_{\mathrm{G}}^{-2 / 3} \approx$ $1.4 M_{\odot}$, where $m_{\mathrm{p}}$ is the proton mass, $\alpha_{\mathrm{G}} \equiv G m_{\mathrm{p}}^{2} /$ $\hbar c=5.9 \times 10^{-39}$ is a dimensionless quantity called gravitational fine-structure constant, and $G$ is the gravitational constant. 


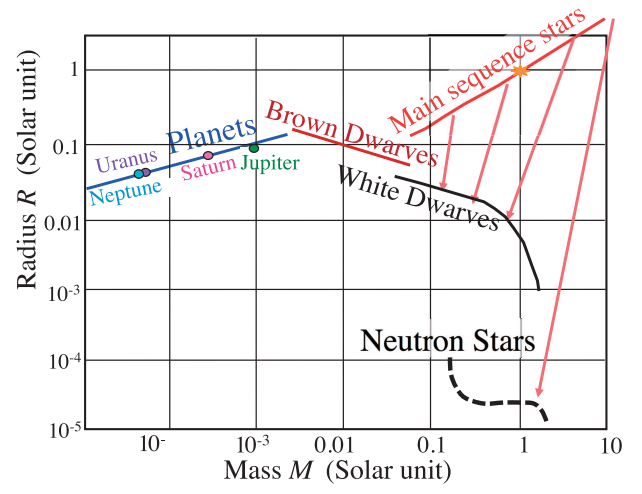

Fig. 1. Mass-radius relations for various types of stars. Downward orange arrows illustrate the evolution of the core of normal stars (omitting their red-giant phase), which involves significant mass loss.

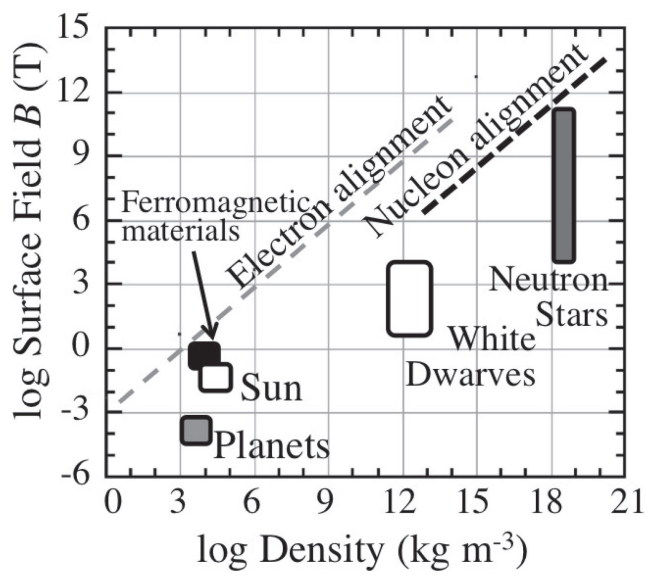

Fig. 2. Typical surface MF intensities of various stars, plotted as a function of their density. Two lines indicate the expected MF intensities, when all electrons (dashed line) or nucleons (dotted line) are spin-aligned to form a ferromagnetic phase. The diagram also incorporates typical ferromagnetic materials.

In Fig. 1, NSs appear as a class with by far the smallest radii. Since they are supported by degenerate pressure of neutrons, the neutron-neutron separation inside them is of the same order as the Compton wavelength of nucleons. As a result, the radius of an NS is $\sim m_{\mathrm{e}} / m_{\mathrm{n}}=1 / 1840$ times that of a white dwarf of the same mass, where $m_{\mathrm{e}}$ and $m_{\mathrm{n}}$ are the electron mass and neutron mass, respectively. The upper-limit mass of NSs is considered to be $\sim 3$ $M_{\odot}$ or less, still with considerable uncertainties.

One interesting aspect of stars is their magnetism, because most of them are magnetized as summarized in Fig. 2. These MFs are in many cases interpreted as due to some electric currents flowing inside a plasma phase, e.g., a metallic-hydrogen core in Jupiter, and an iron-rich molten interior of the Earth. On the other hand, the gross proportionality seen in Fig. 2, between the MF and the density, suggests alternatively that ferromagnetism (of electrons or nucleons) is contributing at least in some objects. In either case, the magnetism is expected to provide valuable information on the EOS. In particular, that of NS may potentially represent some physics in extreme conditions.

The present paper reviews studies of magnetism of $\mathrm{NSs}^{1)}$ performed in the past quarter century mainly with Japanese X-ray satellites, Ginga launched in 1987 February, ${ }^{2)}$ ASCA in 1983 February, ${ }^{3)}$ and Suzaku in 2005 July. ${ }^{4)}$ In addition, we incorporate results from the Monitor of All-sky X-ray Image $(\mathrm{MAXI}){ }^{5)}$ placed onboard the Japan Experimental Module Kibo which comprises the International Space Station. Because of the page limitation, the paper rather poorly covers topics with radio pulsars, and theoretical aspects of the NS interior.

\section{Neutron stars}

2.1. Formation of neutron stars. In a lifetime of $10^{5.5}-10^{7.5}$ years, a massive star reaches an endpoint of its evolution, where it makes a corecollapse supernova explosion. In this dramatic event with a huge energy release $\left(\sim 10^{46} \mathrm{~J}\right)$, an inner part ("core") of the star collapses by its own gravity towards the center, leading to the formation of an NS if the initial mass of the star is about $10-20 M_{\odot}$. By bounced shocks, the remaining outer parts are ejected into the interstellar space to form an expanding shell structure known as a supernova remnant (SNR). If the progenitor star has a larger initial mass, e.g., $>25$ $M_{\odot}$, the infalling core is considered to be heavier than the upper limit for NSs, and hence to collapse into a black hole.

Figure 3 shows the Crab Nebula ${ }^{6}$ ) which exploded in the year of 1054, and its central NS known as the Crab Plusar. It is the best-known example of NS-SNR association, even though it would not be a typical SNR because it lacks the blast-wave heated thermal remnant and has only the nonthermal pulsar-wind nebula. Here, the term "pulsar" generally means an NS of which the radiation intensity exhibits clear periodicity at its rotation period $P$. An NS is not called a pulsar if its $P$ is unknown.

In addition to the above canonical scenario, there can be yet another channel of NS formation, called "accretion induced collapse". ${ }^{7}$ ) When a white dwarf in a binary system gradually gains its mass 
through accretion, it will hit the Chandrasekhar limit and ignite fusion reactions near the center. If the white dwarf has a composition which is rich in $\mathrm{C}$ and $\mathrm{O}$, it will end up with a Type Ia supernova via thermonuclear runaway explosion. In contrast, the white dwarf will collapse into an NS if the composition is mainly of $\mathrm{O}, \mathrm{Ne}$, and $\mathrm{Mg}$, because the electron capture takes over the nuclear burning and reduces the pressure. Details of this process wait for future investigation.

2.2. Major properties of neutron stars. Of all classes of stars, NSs are regarded as champions in three respects; the highest density, the fastest rotation, and the strongest MFs. Yet another characteristic of NSs is their strong gravity, although in this respect they are next to black holes.

Composition: An NS consists mostly of neutrons, and is sustained by their degenerate pressure against its extreme gravity. These neutrons are produced in the collapsing stellar core, via a process known as electron capture or neutronization. That is, a $\beta$-type reaction between two nuclear species $\mathrm{A}(N, Z)$ and $\mathrm{A}(N+1, Z-1)$,

$$
\mathrm{A}(N, Z)+\mathrm{e}^{-} \rightleftharpoons \mathrm{A}(N+1, Z-1)+\nu_{\mathrm{e}},
$$

where $Z$ is the proton number and $N$ is that of neutrons, would not proceed to the right any longer if $N \gg Z$, because $\mathrm{A}(N+1, Z-1)$ would have a larger mass than the left hand side. However, when $\rho$ becomes extremely high in the collapsing stellar core, the Fermi energy of electrons will increase as $\epsilon_{\mathrm{F}}^{(\mathrm{e})} \propto$ $\rho^{1 / 3}$ (relativistic case), and make the rest-mass energy of the left hand side higher than that of the right hand side. Then, the electron to be emitted by $\mathrm{A}(N+1, Z-1)$ has a lower energy than $\epsilon_{\mathrm{F}}^{(\mathrm{e})}$, so that the leftward reaction is forbidden by Pauli's exclusion principle.

Mass and radius: An NS is an extremely tiny object, with a typical mass $M_{\mathrm{NS}}$ and radius $R_{\mathrm{NS}}$ of

$$
M_{\mathrm{NS}} \approx 1.4 M_{\odot}, \quad R_{\mathrm{NS}} \approx 11 \mathrm{~km} .
$$

Their radii are thus $\sim 5$ orders of magnitude (just the atom vs. nucleus size ratio) smaller than the Solar radius. When $M_{\mathrm{NS}}$ is given, we can approximately calculate $R_{\mathrm{NS}}$, by minimizing the overall energy

$$
E_{\mathrm{tot}}=-\frac{3}{5} G \frac{M_{\mathrm{NS}}^{2}}{R_{\mathrm{NS}}}+b p_{\mathrm{d}} V
$$

with respect to $R_{\mathrm{NS}}$. Here, the first term on the right hand side represents self gravity (assuming a uniform density). The second term is the internal energy, where $p_{\mathrm{d}}$ is the degenerate pressure of neutrons, $V$ is the stellar volume, and $b$ is a numerical factor of order unity. In non-relativistic case, the EOS can be approximated as $p_{\mathrm{d}} \sim \epsilon_{\mathrm{F}}^{(\mathrm{e})}\left(\rho / m_{\mathrm{n}}\right)=b^{\prime}\left(h^{2} / 5 m_{\mathrm{n}}\right)(\rho /$ $\left.m_{\mathrm{n}}\right)^{5 / 3}$, with $h$ the Planck constant, and $b^{\prime}$ yet another numerical constant of order unity. Using this EOS, the solution to Eq. [3] is expressed as $R_{\mathrm{NS}} \sim$ $10\left(M_{\mathrm{NS}} / M_{\odot}\right)^{-1 / 3} \mathrm{~km}$.

The actual mass-radius relation of NSs is subject to special and general relativity, and nuclear interactions among neutrons which make the object a strongly-interacting Fermi gas. Because we still have limited knowledge of the nuclear interaction, or the "nuclear EOS" that replaces the above simplest EOS, theoretical $M_{\mathrm{NS}}$ vs. $R_{\mathrm{NS}}$ relations have not yet been accurately identified. Conversely, if we can measure $M_{\mathrm{NS}}$ and $R_{\mathrm{NS}}$ of a fair number of NSs with a sufficient accuracy, the results will give a valuable clue to the nuclear EOS.

Observationally, the values of $M_{\mathrm{NS}}$ measured from about $50 \mathrm{NSs}^{8)}$ are concentrated in a narrow range around Eq. [2], with at most $\pm 30 \%$ scatter: so are the measured values of $R_{\mathrm{NS}}$. On one hand, we need to explain how such narrow distributions are realized. On the other hand, these individual measurements must be made yet with higher accuracies, in order to constrain the nuclear EOS.

High density: Because of the extremely small $R_{\mathrm{NS}}$, NSs are by far the densest objects in the Universe. From Eq. [2], their volume-averaged density becomes $\rho \sim 5 \times 10^{17} \mathrm{~kg} \mathrm{~m}^{-3}$, which is by $>13$ orders of magnitude higher than those of the densest metals, and even exceeds those of atomic nuclei, $\rho_{0}=2.3 \times 10^{17} \mathrm{~kg} \mathrm{~m}^{-3}$. At the center of $\mathrm{NSs}$, the density is expected to become as high as $\sim 10 \rho_{0}$.

Rapid rotation: Among all celestial objects, NSs (except mass-accreting ones) are by far the most rapidly rotating class, with the fastest known record having a rotation period of $P=1.5 \mathrm{msec}$. This feature of NSs is a result of angular momentum inheritance from their progenitor stars, together with their extremely small radii. As a Gedanken experiment, let us compress the Sun into an NS (although the Sun would never evolve so). Then, the Solar rotation period, about a month, would decrease to $P \sim$ $0.5 \mathrm{msec}$ through angular momentum conservation, because the radius should decrease by a factor of $\sim 7 \times 10^{4}$. This happens to be the shortest period that an NS can attain, as it would break up for $P<$ $0.5 \mathrm{msec}$ due to centrifugal force.

Strong magnetic fields: Another outstanding feature of NSs is their strongest MFs among all celestial objects (Fig. 2). As described later in $\S 2.3$, a 


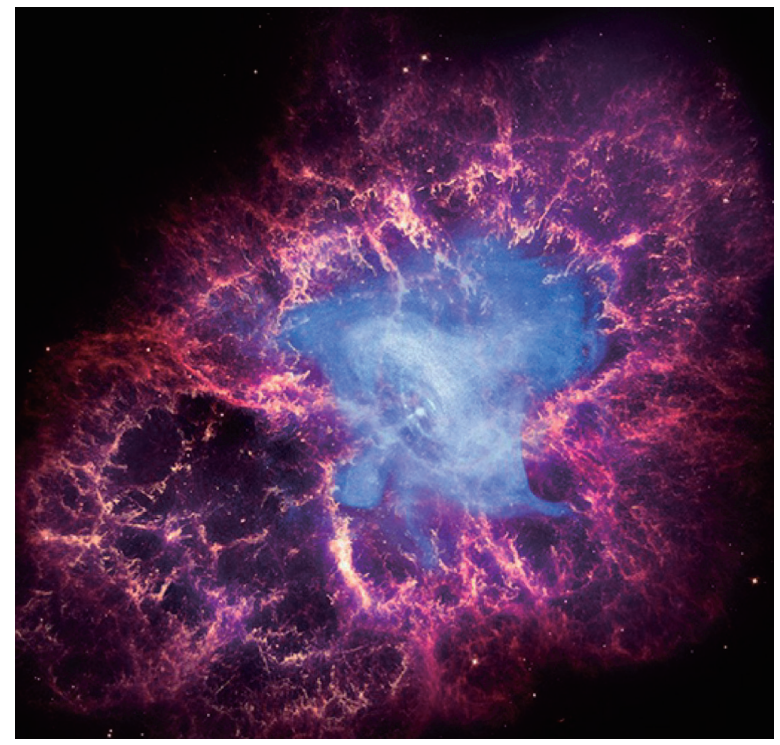

Fig. 3. A composite image of the Crab Nebula, taken in the optical (purple) and X-rays (cyan). The Crab pulsar is seen at the center. The image size is about $6^{\prime} \times 7^{\prime}$. Taken from the Chandra Photo Album.

majority of NSs have $B_{\mathrm{d}} \sim 10^{8} \mathrm{~T}$, where $B_{\mathrm{d}}$ refers to dipole MF intensity (more properly, magnetic flux density) measured at the magnetic poles on the NS surface. It is related to the object's magnetic dipole moment $\mathcal{M}$ as

$$
\mathcal{M}=2 \pi R_{\mathrm{NS}}^{3} B_{\mathrm{d}} .
$$

The above value of $B_{\mathrm{d}}$ can be understood through the same Gedanken experiment as above; a typical Solar dipole field of $B_{\mathrm{d}} \sim 0.02 \mathrm{~T}$ would be compressed, during the core collapse, to $B_{\mathrm{d}} \sim 10^{8} \mathrm{~T}$ through conservation of magnetic flux.

2.3. Classification of neutron stars. Among several ways to classify NSs, a convenient one, shown in Fig. 4, is to use their values of $P$ and $B_{\mathrm{d}}$. There, the most dominant subclass is radio pulsars, i.e., isolated NSs with $P$ of a few tens msec to several sec, and $B_{\mathrm{d}}=10^{7}-10^{9} \mathrm{~T}$. Some of them are found in SNRs, like the case of Fig. 3. Since these NSs are considered to lose their rotational energies by radiating magnetic dipole radiation $(\S 3.1)$, they will move to the right on Fig. 4 as they get older. Their age can be estimated using a quantity called characteristic age, defined as

$$
\tau_{\mathrm{c}} \equiv P / 2 \dot{P}
$$

where $\dot{P}$ is the measured period derivative.

The second subclass in Fig. 4 is binary $\mathrm{X}$-ray pulsars (BXPs) extending horizontally at $B_{\mathrm{d}}=(1-$ $7) \times 10^{8} \mathrm{~T}$. They form binaries with (usually massive)

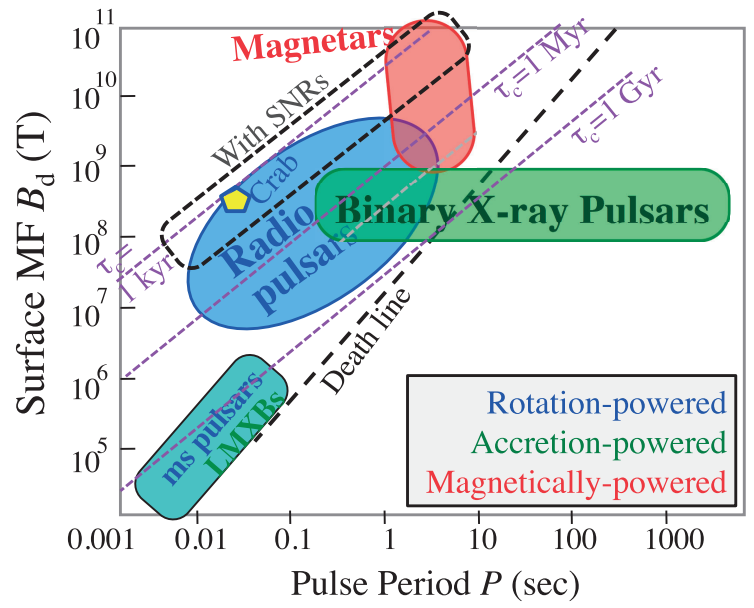

Fig. 4. A schematic classification of NSs, in terms of $P$ and $B_{\mathrm{d}}$. Blue, green, and red indicate rotation-powered, accretionpowered, and magnetically-powered objects, respectively. Grids for $\tau_{\mathrm{c}}$ of Eq. [5] are provided by dashed purple lines. "Death line" means that rotation-powered objects become unable to emit significant radiation when they cross this line. NSs residing in SNRs are also indicated.

companion stars, and capture their stellar winds to emit X-rays with strong pulsations, as the captured matter will fall to the two magnetic poles of the NS where it is shock heated to form X-ray emitting hot "accretion columns". The wide scatter in $P$ seen among BXPs, from $2 \mathrm{~ms}$ to $\sim 10^{4} \mathrm{~s}$, results from their interaction with the accreting matter $(\S 3.2)$.

Yet another subclass is those at the low- $B_{\mathrm{d}}$ and short- $P$ end, a mixture of millisecond pulsars (MSPs; isolated or in wide binaries) and Low-Mass X-ray Binaries (LMXBs). The latter class of NSs accrete matter from their low-mass companion stars, to emit $\mathrm{X}$-rays with weak or almost no pulsation. Usually, these NSs are considered to be old $\left(>10^{9} \mathrm{yr}\right)$.

Finally, a special subclass, located at the highest-MF region of $B_{\mathrm{d}} \sim 10^{10}-10^{11} \mathrm{~T}$, is called "magnetars". In $\S 4$, we present more detailed descriptions of these objects, employing a working hypothesis that the values found in Fig. 4 represent their true surface MFs.

As indicated by Fig. 4, NSs exhibit not only very strong MFs, but also a remarkable scatter in $B_{\mathrm{d}}$ over 8 orders of magnitude. It is not known how the MF can scatter this much, given strong concentrations of the observed $M_{\mathrm{NS}}$ and $R_{\mathrm{NS}}$ around their canonical values [Eq. [2]]. It is unclear, either, whether NSs with strong and weak MFs differ systematically (but within the narrow scatter) in their masses. More fundamentally, we need to clarify how these strong 
MFs are held: is the magnetism driven by some electric currents (electric magnets), or sustained by some kind of ferromagnetism (permanent magnets)?

2.4. Energy sources of neutron stars. The NSs in Fig. 4 all emit electromagnetic radiation in some frequencies, with a typical luminosity of $L=$ $10^{26}-10^{31} \mathrm{~W}$. From the energy sources of this radiation, the NS can be classified in a different manner into the following three categories. There is in fact yet another class, thermally radiating NSs. Although observations of their cooling provide valuable clues to the physical conditions inside NSs, the subject is beyond the scope of the present paper.

Rotation-powered NSs: radio pulsars and MSPs in Fig. 4 emit rather broad-band radiation (mainly radio but sometimes to optical or even higher frequencies) by consuming their rotational energies, which is given as

$$
E_{\mathrm{rot}}=\frac{I}{2}\left(\frac{2 \pi}{P}\right)^{2}=2.0 \times 10^{40} I_{38}\left(\frac{P}{0.3 \mathrm{~s}}\right)^{-2} \mathrm{~J}
$$

where $I_{38}$ is the moment of inertia in units of $10^{38} \mathrm{~kg} \mathrm{~m}^{2}$. By differentiating this equation, the luminosity available via spin down can be written as

$$
\begin{aligned}
L_{\mathrm{rot}} & =-\frac{d E}{d t}=\frac{(2 \pi)^{2} I}{P^{3}} \dot{P} \\
& =1.5 \times 10^{26} I_{38}\left(\frac{P}{0.3 \mathrm{~s}}\right)^{-3}\left(\frac{\dot{P}}{10^{-15} \mathrm{~s} / \mathrm{s}}\right) \mathrm{W} .
\end{aligned}
$$

For reference, the fiducial values of $P=0.3 \mathrm{~s}$ and $\dot{P}=1 \times 10^{-15} \mathrm{~s} \mathrm{~s}^{-1}$ gives, via Eq. [5], $\tau_{\mathrm{c}}=4.8 \mathrm{Myr}$, which is a typical age of radio pulsars. How to convert $E_{\text {rot }}$ into $L_{\text {rot }}$ is explained in $\S 3.1$.

Rotation-powered NSs are a site of acceleration of particles and production of electron-positron pairs. These processes have been extensively studied ${ }^{9), 10)}$ since the discovery of pulsars in $1967 .^{11)}$ Instead of reviewing this subject in details, let us consider a simple exercise; why MSPs, in spite of their much weaker MFs than those of young radio pulsars, can still accelerate particles to very high energies and emit pulsed GeV gamma-rays, as discovered with the Fermi Gamma-Ray Space Telescope. ${ }^{12)}$ The acceleration in rotation-powered NSs is likely to be caused by strong electric fields induced by their rotating MFs, at locations close to the "light cylinder", $R_{\mathrm{LC}}=c P / 2 \pi$, where the velocity of co-rotation with the pulsar reaches $c$. Using the MF strength near $R_{\mathrm{LC}}$, i.e., $B_{\mathrm{LC}} \sim B_{\mathrm{d}}\left(R_{\mathrm{NS}} / R_{\mathrm{LC}}\right)^{3)}$, the induced electric potential therein will be estimated as $c \cdot B_{\mathrm{LC}} \cdot R_{\mathrm{LC}} \sim$ $c B_{\mathrm{d}} R_{\mathrm{NS}}^{3} R_{\mathrm{LC}}^{-2} \propto B_{\mathrm{d}} / P^{2}$. MSPs have some 4 orders of magnitude lower $B_{\mathrm{d}}$ than representative radio pulsars, but typically 2 orders of magnitude shorter $P$, so that the induced potential is similar between these two subclasses. Thus, MSPs can accelerate particles to similarly high energies as radio pulsars, but with a much lower luminosity due to their much smaller volume available for the process.

Accretion-powered NSs: In Fig. 4, BXPs and LMXBs accrete gas from their binary companions, and emit X-rays powered by gravitational energies released by the accreting matter. The energy available by this process is given as

$$
\begin{aligned}
E_{\text {grav }} & =G \frac{M_{\mathrm{NS}} \Delta M}{R_{\mathrm{NS}}} \\
& =3.4 \times 10^{42}\left(\frac{M_{\mathrm{NS}}}{1.4 M_{\odot}}\right)\left(\frac{R_{\mathrm{NS}}}{11 \mathrm{~km}}\right)^{-1}\left(\frac{\Delta M}{10^{-4} M_{\odot}}\right) \mathrm{J}
\end{aligned}
$$

where $\Delta M$ is the overall mass accreted on the NS. Then, using the accretion rate $\dot{M} \equiv \Delta M /$ (accretion lifetime) which is typically of the order of $\dot{M} \sim$ $10^{-4} M_{\odot} / 10^{7} \mathrm{yr}=0.6 \times 10^{12} \mathrm{~kg} \mathrm{~s}^{-1}$, the luminosity is given as

$$
L_{\text {grav }}=1.7 \times 10^{28} \dot{M} /\left(1 \times 10^{12} \mathrm{~kg} \mathrm{~s}^{-1}\right) \mathrm{W}
$$

where the dependence on $M_{\mathrm{NS}}$ and $R_{\mathrm{NS}}$ was omitted for simplicity.

This $L_{\text {grav }}$ has a clear upper limit, called Eddington limit, which is given as

$$
\begin{aligned}
L_{\mathrm{Edd}} & =4 \pi c G M_{\mathrm{NS}} m_{\mathrm{p}} f_{\mathrm{b}} / \sigma_{\mathrm{T}} \\
& =2.1 \times 10^{31}\left(M_{\mathrm{NS}} / 1.4 M_{\odot}\right)\left(f_{\mathrm{b}} / 1.16\right) \mathrm{W}
\end{aligned}
$$

where $\sigma_{\mathrm{T}}=6.65 \times 10^{-29} \mathrm{~m}^{-2}$ is the Thomson cross section, and $f_{\mathrm{b}}$ is baryon-to-electron number ratio which is 1 for Hydrogen, 2 for Helium and heavier elements, while 1.16 for matter with the cosmic abundance. Above this luminosity, the radiation pressure working on electrons exceeds the gravity working on baryons, and blow off the matter.

When $L_{\text {grav }}$ of Eq. [9] is emitted isotropically from the whole NS surface as blackbody radiation of temperature $T_{\mathrm{bb}}$, the Stefan-Boltzmann law, together with Eq. [2], gives

$$
T_{\mathrm{bb}}=\left(\frac{L_{\text {grav }}}{4 \pi R_{\mathrm{NS}}^{2} \sigma}\right)^{1 / 4}=1.5 \times 10^{7}\left(\frac{L_{\text {grav }}}{L_{\mathrm{Edd}}}\right)^{1 / 4} \mathrm{~K}
$$

where $\sigma=5.67 \times 10^{-8} \mathrm{~W} \mathrm{~m}^{-2} \mathrm{~K}^{-4}$ is the StefanBoltzmann constant. Thus, the heated NS surface has a temperature just corresponding to X-ray energies.

Magnetically-powered NSs: As detailed later in $\S 4$, objects of the magnetar class in Fig. 4 radiate mainly in X-rays. However, they cannot be rotation- 
powered objects, since their X-ray luminosities largely exceed $L_{\text {rot }}$ of Eq. [7]. As discussed in $\S 4$, they are not likely to be accretion-powered objects, either. Instead, magnetars are considered to be magnetically-powered NSs, which emit X-rays by consuming their magnetic energy given as

$$
\begin{aligned}
E_{\mathrm{mag}} & =\left(\frac{B_{\mathrm{d}}^{2}}{2 \mu_{0}}\right)\left(\frac{8 \pi}{3} R_{\mathrm{NS}}^{3}\right) \\
& =4.4 \times 10^{40}\left(\frac{B_{\mathrm{d}}}{10^{11} \mathrm{~T}}\right)^{2} \mathrm{~J}
\end{aligned}
$$

where $\mu_{0}$ is the vacuum magnetic permeability.

\section{Measurements of magnetic fields of neutron stars}

In this section, we describe three representative ways to estimate the MFs of NSs. The first one is for isolated NSs, while the latter two are applicable to accreting NSs. We have contributed to the third method, and partially to the second one as well.

3.1. Pulse periods and period changes. The most widely used way to estimate $B_{\mathrm{d}}$ of isolated NSs is to assume that their spin-down energy release, $L_{\text {rot }}$ of Eq. [7], goes into magnetic dipole radiation, ${ }^{9)}$ of which the luminosity is written as

$$
L_{\mathrm{M} 2}=\frac{(2 \pi / 3)\left(B_{\mathrm{d}} R^{3} \sin \theta\right)^{2}}{c^{3} \mu_{0}}\left(\frac{2 \pi}{P}\right)^{4}
$$

where $\theta$ is the angle between the rotational and magnetic axes. We can equate this with Eq. [7], to obtain $B_{\mathrm{d}} \sin \theta \propto \sqrt{P \dot{P}}$. Using Eq. [2], the relation can be expressed more explicitly as

$$
B_{\mathrm{d}} \sin \theta=0.32 \times 10^{8}\left(\frac{P}{0.3 \mathrm{~s}}\right)^{1 / 2}\left(\frac{\dot{P}}{10^{-15} \mathrm{~s} / \mathrm{s}}\right)^{1 / 2} \mathrm{~T} .
$$

This " $P-\dot{P}$ technique" has yielded the values of $B_{\mathrm{d}}$ of all non-accreting NSs in Fig. 4, including radio pulsars, MSPs, and magnetars. In particular, it yields $B_{\mathrm{d}} \sin \theta=2.8 \times 10^{8} \mathrm{~T}$ for the Crab pulsar, which has $P=0.0332 \mathrm{sec}, \dot{P}=4.22 \times 10^{-13} \mathrm{~s} \mathrm{~s}^{-1}$, and $L_{\mathrm{rot}}=$ $5.1 \times 10^{31} \mathrm{erg} \mathrm{s}^{-1}$ employing $I=1.1 \times 10^{38} \mathrm{~kg} \mathrm{~m}^{2}{ }^{13)}$ While this method is convenient, it gives only a lower limit on $B_{\mathrm{d}}$ in the sense that $\theta$ is usually unknown. At the same time, it may be regarded as giving an upper limit, because the spin-down may take place via other energy output channels, including acceleration of particles via unipolar induction mechanism $(\S 2.4),{ }^{10}$ and creation of plasma outflows. Thus, the estimates with Eq. [14] are considered rather crude. These additional energy losses are considered to have very similar strengths and functional forms to Eq. [13], ${ }^{10)}$ but without the $\sin \theta$ dependence. Therefore, the spin-down behavior may not depend on $\theta$ as strongly as implied by Eq. [13].

By solving Eq. [14] under a constant $B_{\mathrm{d}}$, the pulse period and luminosity are predicted to evolve as a function of time $t$ as

$$
\begin{aligned}
& P(t)=P_{0}\left(1+t / t_{1}\right)^{1 / 2}, \\
& E_{\mathrm{rot}}(t)=E_{\mathrm{rot}}(0)\left(1+t / t_{1}\right)^{-1}, \\
& L_{\mathrm{rot}}(t)=L_{\mathrm{M} 2}(t)=L_{0}\left(1+t / t_{1}\right)^{-2},
\end{aligned}
$$

where subscript 0 specifies the value at the birth $(t=0)$, and $t_{1}$ is a time constant over which the initial rotational energy is halved. From this $P(t)$, the characteristic age of Eq. [5] can be expressed as

$$
\tau_{\mathrm{c}}(t)=t+t_{1} .
$$

Thus, $\tau_{\mathrm{c}}$ can be identified with the true age $t$ when $t_{1}$ can be neglected. Indeed, the Crab Pulsar has $\tau_{\mathrm{c}}=1250 \mathrm{yr}$, which is sufficiently close to its true age, $962 \mathrm{yr}$ as of 2016. Furthermore, by integrating the above $L_{\text {rot }}$ from $t=0$ to $t=+\infty$, we obtain

$$
E_{\text {rot }}(0)=L_{0} t_{1} \text {. }
$$

Combining this with Eq. [13] and Eq. [6], we obtain

$$
t_{1} \sim 7 \times 10^{3}(P / 10 \mathrm{~ms})^{2}\left(B_{0} \sin \theta / 10^{8} \mathrm{~T}\right)^{-2} \mathrm{yr}
$$

where $P_{0} \sim 10 \mathrm{msec}$ is assumed. Thus, magnetars with $B_{0} \sim 10^{11} \mathrm{~T}$ would lose half the initial rotational energy in only a few days, while NSs with $B \sim 10^{5} \mathrm{~T}$ would have $t_{1} \sim 10^{10} \mathrm{yr}$, i.e., a cosmological time scale.

3.2. Accretion torque. To estimate MFs of mass-accreting NSs (those in binaries), which exhibit in Fig. 4 a large scatter in $P$, we must consider accretion torque, namely, angular momentum transfer to/from the accreting matter. In this case, the NS can either spin up or spin down, unlike Eq. [13] which always acts to spin down the NS.

The accretion torque formalism was developed in the late 1970's, ${ }^{14)}$ and is being calibrated and confirmed through MAXI monitoring of several BXPs including GX 304-15) and $4 \mathrm{U}$ 1626-67'16) in particular. It assumes that the gravitational pull working on the accreting matter becomes counterbalanced by the magnetic presssure at a radius $R_{\mathrm{A}}$, called Alfvén radius, which can be expressed as

$$
\begin{aligned}
R_{\mathrm{A}}= & 10^{6} \zeta\left(\frac{L_{\mathrm{x}}}{10^{30} \mathrm{~W}}\right)^{-2 / 7}\left(\frac{B_{\mathrm{d}}}{10^{8} \mathrm{~T}}\right)^{4 / 7} \\
& \times\left(\frac{R_{\mathrm{NS}}}{11 \mathrm{~km}}\right)^{10 / 7}\left(\frac{M_{\mathrm{NS}}}{1.4 M_{\odot}}\right)^{1 / 7} \mathrm{~m}
\end{aligned}
$$


where $\zeta$ is a numerical factor of order unity, and $L_{\mathrm{x}} \propto \dot{M} \propto 4 \pi R_{\mathrm{A}}^{2} \rho v_{\mathrm{ff}}$ is the X-ray luminosity with $v_{\mathrm{ff}}$ being the radial free-fall velocity at $R_{\mathrm{A}}$. This $L_{\mathrm{x}}$ is essentially the same as $L_{\text {grav }}$ of Eq. [9]. At radii $r>R_{\mathrm{A}}$, the matter is assumed to rotate with the Keplerian angular frequency as

$$
\omega_{\mathrm{K}}(r)=\sqrt{G M_{\mathrm{NS}} / r^{3}} .
$$

At $r<R_{\mathrm{A}}$, in contrast, the matter is assumed to be captured by the MF lines and fall to the accretion columns with $v_{\mathrm{ff}}$. The NS will receive momentum (positive torque) from the matter, and hence be spun up, if it is rotating slowly as $\Omega<\omega_{\mathrm{K}}\left(R_{\mathrm{A}}\right)$, where $\Omega \equiv P / 2 \pi$ is the angular frequency of the NS rotation. (We implicitly assume that the accretion plane is nearly perpendicular to the pulsar's spin axis and the matter rotates in the same direction as the pulsar.) In contrast, a fast rotator with $\Omega>\omega_{\mathrm{K}}\left(R_{\mathrm{A}}\right)$ will give angular momentum to the accreting matter, and expel it out. This "propeller effect" will spin-down the NS.

Most of accreting NSs, including both BXPs and LMXBs, are expected to be in a condition of near torque equilibrium, namely, $\Omega \approx \omega_{\mathrm{K}}\left(R_{\mathrm{A}}\right)$, because their spin up/down time scales due to the accretion torque are estimated to be shorter than their life times. Then, expressing the $\Omega \approx \omega_{\mathrm{K}}\left(R_{\mathrm{A}}\right)$ condition using Eq. [21] and Eq. [22], we obtain so-called equilibrium rotation period as

$$
P_{\text {eq }} \sim 0.6 \zeta^{3 / 2}\left(\frac{L_{\mathrm{x}}}{10^{30} \mathrm{~W}}\right)^{-3 / 7}\left(\frac{B_{\mathrm{d}}}{10^{8} \mathrm{~T}}\right)^{6 / 7} \mathrm{sec}
$$

where the dependence on $M_{\mathrm{NS}}$ and $R_{\mathrm{NS}}$ has again been suppressed. Thus, accreting NSs with stronger MFs and lower luminosities are expected to have longer pulse periods. Conversely, by measuring $P$ and $L_{\mathrm{x}}$ of an accreting NS, and equating $P$ with $P_{\text {eq }}$, we can estimate its $B_{\mathrm{d}}$ as

$$
B_{\mathrm{d}} \sim 1.8 \times 10^{8} \zeta^{-7 / 4}\left(\frac{L_{\mathrm{x}}}{10^{30} \mathrm{~W}}\right)^{1 / 2}\left(\frac{P}{1 \mathrm{sec}}\right)^{7 / 6} \mathrm{~T} .
$$

In Fig. 4, the MF strengths of LMXBs have been estimated in this manner, using their fast spins and high luminosities, although the results are rather uncertain, reflecting uncertainties, e.g., in the numerical factor $\zeta$ in Eq. [21].

3.3. Electron cyclotron resonances. Among accreting NSs, BXPs $(\S 2.3$; extending horizontally in Fig. 4) have typically $B_{\mathrm{d}} \sim 10^{8} \mathrm{~T}$, and allow particularly accurate measurements of $B_{\mathrm{d}}$ based on electron cyclotron resonances. In fact, the MF strengths of these objects have all been determined in this way.
This technique utilizes the basic physics that an electron in a uniform magnetic field $B$ makes cyclotron gyration at an angular frequency of $\Omega=e B / m_{\mathrm{e}}$, which translates to a photon energy of

$$
E_{\mathrm{a}}=\hbar e B_{\mathrm{d}} / m_{\mathrm{e}}=11.6\left(B_{\mathrm{d}} / 10^{8} \mathrm{~T}\right) \mathrm{keV}
$$

ignoring special and general relativistic effects. In terms of quantum mechanics, this is identical to the Landau level intervals. As a result, an X-ray spectrum from a BXP often bears a noticeable feature, called Cyclotron Resonance Scattering Feature (CRSF) $\left.\left.{ }_{17}\right), 18\right)$ at the energy of Eq. [25] and its higher harmonics; Fig. 5 shows two examples. Evidently, detections of CRSFs provide the most direct measurements of the surface dipole MFs of BXPs through Eq. [25]. However, by the mid 1980's, only two examples of CRSF were known; Her X-1 ${ }^{19}$ ) at $E_{\mathrm{a}}=35 \mathrm{keV}$ and $4 \mathrm{U} 0115+63^{20)}$ at $E_{\mathrm{a}}=11 \mathrm{keV}$.

Using Ginga launched in $1987,{ }^{2)}$ we have drastically enlarged the number of CRSF detections. Beginning with a discovery at $E_{\mathrm{a}}=21 \mathrm{keV}$ from $4 \mathrm{U}$ 1538-52, ${ }^{21)}$ CRSFs have been detected one after another, from $\mathrm{X} 0331+53$ at $28 \mathrm{keV},{ }^{22)} \mathrm{Cep} \mathrm{X-4} \mathrm{at}$ $29 \mathrm{keV},{ }^{23)} 4 \mathrm{U} 1907+09$ at $\left.20 \mathrm{keV},{ }^{17}\right) \mathrm{GX} 301-2$ at $36 \mathrm{keV},{ }^{17}$ ) and Vela X-1 at 25 (or 50) keV. ${ }^{17}$ ) In addition, the previously known two CRSFs in Her $\mathrm{X}-1^{24)}$ and $4 \mathrm{U} 0115+63^{25)}$ were reconfirmed. These results have established that CRSFs are a common feature of BXPs, and provide their surface MFs in the most reliable way. The measured values of $E_{\text {a }}$ were apparently concentrated in the range of $10-40 \mathrm{keV}$, which implies $B_{\mathrm{d}}=(1-4) \times 10^{8} \mathrm{~T}$ from Eq. [25]. Although CRSFs can theoretically appear either in absorption or emission, and the Her X-1 result was at first interpreted as an emission line, ${ }^{19)}$ these Gingadetected CRSFs, including that of Her X-1, were all found in absorption.

Through these observations, we showed that the BXP spectra with a CRSF can be described, as a function of the X-ray energy $E$, as ${ }^{17), 18), 21), 26)}$

$$
f(E)=\xi(E) \times f_{\mathrm{NPEX}}(E) \times \Psi(E) .
$$

Here, $\xi(E)$ is a factor for photoelectric absorption, and

$$
f_{\mathrm{NPEX}}(E)=\left(A_{1} E^{-\Gamma_{1}}+A_{2} E^{+\Gamma_{2}}\right) \exp \left(-\frac{E}{E_{\text {cut }}}\right)
$$

is a continuum model called "NPEX" (Negative and Positive power-law with EXponential cutoff), ${ }^{17), 26)}$ with $A_{1}, A_{2}, \Gamma_{1}, \Gamma_{2}$, and $E_{\text {cut }}$ all positive free parameters. The first term $E^{-\Gamma_{1}} \exp \left(-E / E_{\text {cut }}\right)$ represents unsaturated Comptonization within hot 
(a) $\mathrm{X} 0331+53$ with $R X T E$

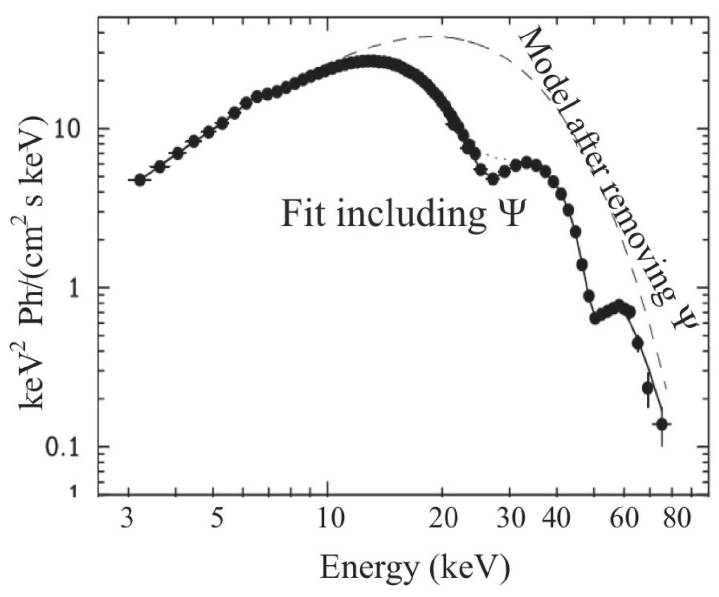

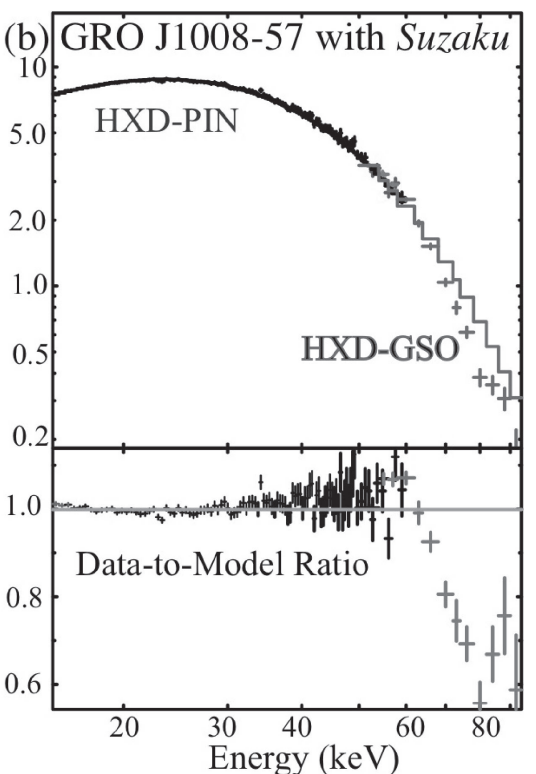

Fig. 5. Examples of X-ray spectra (in $\nu F \nu$ presentation) of two transient BXPs with CRSFs. (a) Deep harmonic CRSFs at 26 and $52 \mathrm{keV}$, observed from X0331+53 with $R X T E^{33)}$ The best-fit model of Eq. [26] with double $\Psi$ is superposed onto the data in a solid line, while the model after removing the factor $\Psi$ is indicated by a dashed curve. (b) A hard X-ray spectrum of GRO J1008-57, obtained with the HXD onboard Suzaku. Histograms in the top panel show a fit with Eq. [26], but without the $\Psi$ factor, while the bottom panel shows the data-to-model ratio. Prominent negative residuals at $\sim 80 \mathrm{keV}$ indicate the highest-energy CRSF observed to date. $^{38)}$

plasmas in the accretion columns of BXPs, while the 2nd term $E^{+\Gamma_{2}} \exp \left(-E / E_{\text {cut }}\right)$, often with $\Gamma_{2}=2.0$ fixed, describes Wien hump produced by the Comptonization. Finally, a multiplicative factor

$$
\Psi(E)=\exp \left\{-\frac{D\left(W E / E_{\mathrm{a}}\right)^{2}}{\left(E-E_{\mathrm{a}}\right)^{2}+W^{2}}\right\}
$$

models the CRSF after the classical cross section of cyclotron resonance, where $D$ is the depth and $W$ is the width of the resonance. This model typically yields $D=0.3-1.5$ and $W=(0.2-0.3) \times E_{\mathrm{a}}$. The latter may reflect various effects in the accretion column, including thermal Doppler effects, MF intensity gradients, Compton scattering, and even induced emission ${ }^{27)}$ as a speculative possibility. We also found clear positive correlation between $E_{\mathrm{a}}$ and $E_{\text {cut. }}$. This suggests that the electron temperature of the accretion column, approximated by $E_{\text {cut }}$ in Eq. [27], is determined mainly by cyclotron cooling. ${ }^{26)}$

Following the Ginga achievements, observations with the BeppoSAX, RXTE, and INTEGRAL satellites increased the number of detected CRSFs. ${ }^{28), 29)}$ From a fair number of these objects, higher harmonic absorptions were detected (e.g., Fig. 5a), up to the 4th harmonic in the most extreme case. $^{30)}$ Another interesting effect which we discovered with Ginga and RXTE is luminosity-dependent changes (up to $\sim 50 \%$ ) in the resonance energy in a few BXPs. ${ }^{31)-33)}$ Possibly the standing shocks which form in the accretion columns change their heights as $\dot{M}$ varies, so that the value of $B_{\mathrm{d}}$ changes to some extent under the dipolar field configuration.

Suzaku, ${ }^{4)}$ launched in 2005, allowed us to search CRSFs in the 30-100 keV energy range, which had poorly been explored by previous missions. Thanks to the Hard X-ray Detector (HXD) ${ }^{34)}$ onboard, this attempt has been successful, as we detected CRSFs from $\mathrm{A} 0535+26$ at $\sim 45 \mathrm{keV},{ }^{35)}$ from GX $304-1$ at $50-54 \mathrm{keV},{ }^{36)}$ from $1 \mathrm{~A} 118-61$ at $\sim 55 \mathrm{keV},{ }^{37)}$ and the highest record of $E_{\mathrm{a}} \sim 76 \mathrm{keV}$ from GRO J100857. ${ }^{38)}$ Although some of these CRSFs had been suggested by previous studies, the Suzaku detections are much more convincing. These new results somewhat expanded the MF distribution of BXPs towards the higher side, to $E_{\mathrm{a}}=10-80 \mathrm{keV}$ and hence

$$
B_{\mathrm{d}}=(1-7) \times 10^{8} \mathrm{~T} \text {. }
$$

In addition, a possible cyclotron emission feature was obtained from $4 \mathrm{U} 1626-67 .{ }^{39)}$

Using Suzaku, we also discovered that 4U 182237, which has a low-mass companion and was so far classified as an LMXB, is in reality a BXP with $E_{\mathrm{a}} \sim 33 \mathrm{keV} .{ }^{40)}$ Including these and other firm examples, the CRSF has been detected to date from 
about 20 objects, out of the $\sim 50$ BXPs that are securely catalogued in the Local Universe. They are subdivided into about 10 objects with Be-type primary stars, about 7 objects capturing stellar winds from their OB-type companions, and 3 examples (Her $\mathrm{X}-1,4 \mathrm{U}$ 1626-67 and 4U 1822-37) accreting from their low-mass companions presumably via Rochelobe overflow.

3.4. Distributions of MF strengths. Figure 6 shows distribution histograms of the values of $B_{\mathrm{d}}$ of NSs, determined with the $P-\dot{P}$ method $(\S 3.1)$ and the CRSF technique ( $\S 3.3)$. There, MF determinations using the accretion-torque method of Eq. [24] are not included, because of large uncertainties involved. However, we are left with a freedom of adjusting the value of $\zeta$ in Eq. [21]. Empirically, we found that $\zeta \sim 1$ can consistently explain the behavior of disk-fed short-period $(P<100 \mathrm{sec})$ BXPs, while $\zeta \sim 5$ is favored by the data of long-period $(P>100 \mathrm{sec})$ objects. $^{41)}$ By thus calibrating $\zeta$, the accurately measured MF strengths of the CRSFbearing BXPs have been reproduced with Eq. [24] within a factor of a few. In this sense, the two independent measurements of $\mathrm{MF}$ of BXPs are consistent with each other.

Figure 6 is approximately equivalent to the projection of Fig. 4 onto the $B_{\mathrm{d}}$ axis. It exhibits three broad peaks; one at $B_{\mathrm{d}}=10^{7}-10^{9} \mathrm{~T}$ covering BXPs and the majority of radio pulsars, another for magnetars at $B_{\mathrm{d}}=10^{10}-10^{11} \mathrm{~T}$, and the other at $B_{\mathrm{d}}=10^{4}-10^{5} \mathrm{~T}$ for MSPs (and LMXBs). It is important to search for clues to this wide scatter in the MF strength, and to the origin of the three apparent peaks. Another interesting point in Fig. 6 is that the MF distribution of BXPs (in green), determined with the CRSF technique via Eq. [29], is much narrower than the main peak formed by radio pulsars. At present, it is unclear whether the two distributions are different, or can be consistent if considering various selection effects.

So far, the search for CRSFs have been unsuccessful on more than half the known BXPs. Some of these objects may have $B_{\mathrm{d}}>7 \times 10^{8} \mathrm{~T}$, and hence exhibit CRSFs in higher energies (e.g., $>80 \mathrm{keV}$ ) than was explored with Suzaku. Promising candidates include BXPs with long pulse periods $\left(P>10^{3} \mathrm{~s}\right)^{41)}$ as inferred from Eq. [24], and/or with very hard spectra as suggested by the $E_{\text {a }}$ vs. $E_{\text {cut }}$ correlation (§3.3). This pertains to the issue of whether the apparent gap in the MF distribution between BXPs and magnetars is intrinsic or due to selection effects.

\section{Challenging the mysteries of magnetars}

4.1. Basic properties. We have now come to the stage of describing magnetars, by far the most enigmatic of all NS subclasses. Historically, the concept of magnetars emerged from two populations of high-energy objects. One is Soft Gamma Repeaters, known since 1979, which emit sporadic gamma-ray bursts with softer spectra than the classical (hence cosmological) gamma-ray burst sources. The other is Anomalous X-ray Pulsars, peculiar X-ray pulsars without binary evidence, of which several were known from the 1980's. Based on observations with, e.g., $A S C A^{3), 42)}$ launched in 1993, these two populations have gradually been found to represent two different aspects of the essentially identical population, and they have come to be collectively called "magnetars". 43),44)

At present, about 30 magnetars are known in the Milky Way and the Magellanic clouds. Furthermore, new magnetars are being discovered typically one per year or so, mainly by the Swift mission which detects short bursts from (re-)activated transient magnetars. Including both these transients and persistent sources, major observational properties of magnetars are summarized as follows.

1. Some magnetars are persistent X-ray sources with a luminosity of $L_{\mathrm{x}}=10^{27}-10^{28} \mathrm{~W}$, while others are transients which are much dimmer during quiescence. They are all very dim in other wavelengths.

2. During active periods, magnetars emit sporadic bursts lasting for $\sim 0.1$ to $\sim 10^{3}$ sec. The burst energy scatters widely over $10^{30}-10^{39} \mathrm{~J}$, and the emission often extends to soft gamma-ray energies.

3. Several magnetars reside near the center of SNRs.

4. All magnetars show clear X-ray pulsations due to their spin, at periods which cluster in a narrow range of $P=2-12$ sec. They also show rather high spin-down rates as $\dot{P} \sim 10^{-13}$ to $10^{-11} \mathrm{~s} \mathrm{~s}^{-1}$.

5. None of them show evidence for binary companions or mass accretion.

6. Compared to those of typical BXPs (e.g., Fig. 5), their persistent spectra in the $1-10 \mathrm{keV}$ range are often very soft, typically equivalent to a blackbody temperature of $\sim 5 \times 10^{6} \mathrm{~K}$.

From item 4 above, magnetars are considered as magnetized NSs, and are rather young from item 3, in spite of their long $P$ (item 4); in fact, Eq. [5] gives 
$\tau_{\mathrm{c}}=1-100 \mathrm{kyr}$. From items 5 and 6 , they are unlikely to be accretion-powered NSs. Furthermore, they cannot be rotation-powered objects, either, since their long $P$ makes $E_{\text {rot }}$ much lower than those of radio pulsars, and hence their $L_{\text {rot }}$ of Eq. [7] falls, in spite of the large $\dot{P}$ (item 4 ), 1-3 orders of magnitude below the observed $L_{\mathrm{x}}$ (item 1$)$. At the same time, Eq. [14] indicates that magnetars have extremely strong $\mathrm{MF}$ as $B_{\mathrm{d}}=10^{10}-10^{11} \mathrm{~T}$, which exceeds the

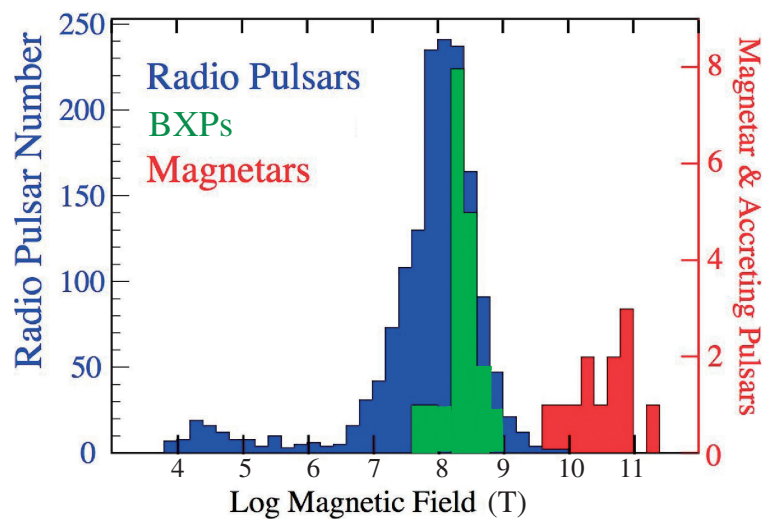

Fig. 6. Distribution histograms of the surface dipole MF, $B_{\mathrm{d}}$, of NSs. Blue indicates radio pulsars and MSPs, and red shows magnetars, both utilizing Eq. [14]. Green shows BXPs with confirmed CRSFs, for which Eq. [25] is utilized. The blue histograms refer to the left ordinate, while the red and green ones to the right. The data refer to the ATNF Pulsar Catalogue, ${ }^{76)}$ available online at http://www.atnf.csiro.au/research/pulsar/ psrcat for updated versions. quantum critical field $B_{\mathrm{c}}=\left(m_{\mathrm{e}} c\right)^{2} / \hbar e=4.4 \times 10^{9} \mathrm{~T}$ at which the energy of Eq. [25] reaches $m_{\mathrm{e}} c^{2}$. As a result, magnetars have been regarded as magnetically-powered NSs $(\S 2.4) .{ }^{43)}$ This is supported by the fact that the values of $B_{\mathrm{d}}$ of magnetars calculated via Eq. [13] clearly decrease as a function of $\tau_{\mathrm{c}}$ of Eq. [5], as we see later in Fig. 12. Then, item 2 can be explained as sudden releases of magnetic energies, $\left.{ }^{43}\right)$ either inside or outside the stars. Finally, items 1 and 6 can be understood by presuming that magnetars emit soft X-rays as thermal emission from the NS surface heated by these magnetic activities.

Besides the above standard scenario, there are alternative explanations of magnetars, including the idea that they are fed by accretion from fossil disks. ${ }^{45}$ However, this possibility is less likely, because the characteristic two-component spectra of magnetars (Fig. 7b) are not observed from BXPs (cf. Fig. 5), even when they become rather $\operatorname{dim}^{35)}$ down to the typical luminosity of magnetars $(<$ a few times $\left.10^{28} \mathrm{~W}\right)$.

The strong-MF interpretation of magnetars ${ }^{43), 44)}$ has thus been generally successful. Nevertheless, it still remains a sort of conjecture, to be called magnetar hypothesis, and leaves us with a number of challenges. First of all, it must be proven whether magnetars really harbor such strong MFs, and are indeed powered by magnetic energies. Even if these basic questions are answered affirmatively, we must clarify how the magnetic energies are consumed to
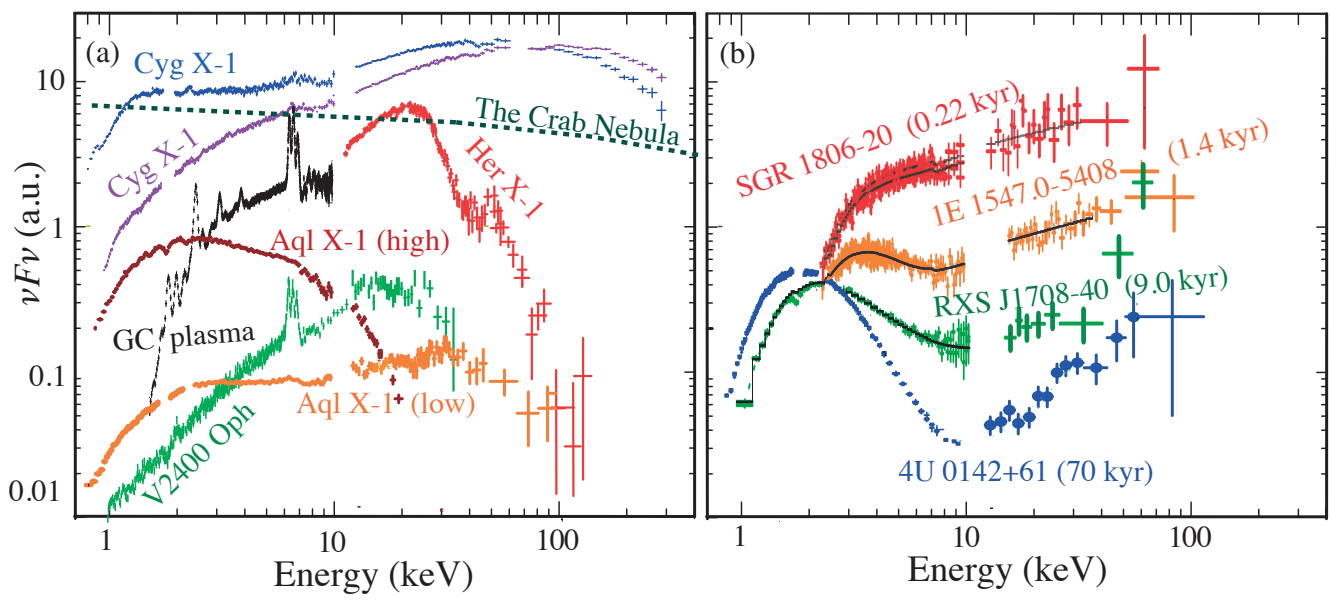

Fig. 7. (a) Suzaku spectra of various Galactic X-ray sources, in their $\nu F \nu$ forms. The $<10 \mathrm{keV}$ and $>10 \mathrm{keV}$ data are from the XIS and the HXD, respectively. Cyg X-1 is an accreting black hole, Her X-1 is a BXP with a CRSF at 35 keV, Aql X-1 is an LMXB, V2400 Oph is an accreting white dwarf, "GC plasma" with strong emission lines represents thin-thermal emission from diffuse hot plasmas surrounding our Galactic center, and the Crab Nebula (in its model form rather than real data) refers to synchrotron emission from the entire nebula shown in Fig. 3. Vertical positions of these spectra are arbitrary. (b) Suzaku spectra of four magnetars in the same form as panel (a), and all normalized at $2.0 \mathrm{keV}$. The number in parenthesis after the name indicates $\tau_{\mathrm{c}}$ of Eq. [5]. 
power the persistent and burst emissions, and what is the relation between these two X-ray emission channels. It also needs to be answered whether the apparent gap in the MF distribution in Fig. 4 between BXPs and magnetars are real or due to some selection effects, and what is special about the formation scenario of magnetars compared to those of NSs of the other subclasses. Hoping to obtain clues to these questions, we have been studying magnetars with Suzaku.

4.2. Emission spectra. Just before the launch of Suzaku, a European group used INTEGRAL to discover that a fair fraction of magnetars emit a separate hard X-ray component, ${ }^{46)}, 47$ ) in addition to the well known soft X-rays (item 6 in $\S 4.1$ ). This novel spectral component has such a hard slope, approximated by a power-law of photon index $\Gamma \sim 1$, that it cannot be readily explained in terms of ordinary high-energy radiation processes, such as synchrotron radiation, inverse Compton scattering, or (non-)thermal Bremsstrahlung.

Our magnetar study with Suzaku utilizes its wide-band capability, realized by the $\mathrm{HXD}^{34)}$ and the X-ray Imaging Spectrometer (XIS). Figure 7a shows a compilation of Suzaku spectra of representative Galactic X-ray sources, while Fig. 7b presents those of 4 magnetars. Thus, we have reconfirmed the reported two-component spectral characteristic of magnetars, because the spectra in panel (b) all exhibit very hard emission rising from $\sim 10 \mathrm{keV}$ towards higher energies, in addition to the long known soft component (item 6 in $\S 4.1$ ). This property is generally not seen in other known classes of compact $\mathrm{X}$-ray sources [e.g., those presented in panel (a)], except young rotation-powered pulsars of which the X-ray spectrum often consists of a soft thermal component and a hard non-thermal tail. ${ }^{48)}$ However, the hard component of these objects usually have $\Gamma \sim 2$, not as hard as those of magnetars. Thus, the unusual spectral shape alone would considerably reinforce the peculiar nature of magnetars.

In Fig. 7b, the hard component of the four magnetars are seen to become weaker (relative to the soft component), but harder in slope, as the objects get older. This is an important discovery by Enoto et al. $(2010)^{49)}$ of a clear evolution in the magnetar spectra, which suggests as if there is a pivot point at several hundreds $\mathrm{keV}$. We have hence proposed a new explanation of the hard component, that it may be due to energy degradation of some energetic input gamma-rays, possibly the $511 \mathrm{keV}$ annihilation photons, rather than a result of successive boosting- up of low-energy electrons/photons towards higher energies. ${ }^{49)}$ Details are explained below.

While magnetars have 2 orders of magnitude slower spins than typical radio pulsars, they have 2 orders of magnitude higher MF. Therefore, the induced electric field in their magnetosphere up to $R_{\mathrm{LC}}$ should be comparable to those in pulsars. Then, as discussed in $\S 2.4$, electrons in the magnetars' magnetosphere will be accelerated and emit abundant gamma-rays (via, e.g., curvature radiation) which in turn produce electron-positron pairs. However, these particles would not freely escape out from magnetosphere, because the centrifugal force of magnetars is $\sim 4$ orders of magnitude weaker than that of pulsars. Then, the particles will fall onto the magnetar surface, where the positrons annihilate to emit $511 \mathrm{keV}$ gamma-rays. In radio pulsars, these photons would again initiate electron-positron cascades. However, in magnetars with $B_{\mathrm{d}}>B_{\mathrm{cr}}$, an energetic photon has a finite probability of colliding with the MF that acts as a virtual photon, and splitting into two real photons. By a repetition of this "photon splitting" process, the input gamma-rays will increase in number, and decrease in individual photon energy, thus forming a hard X-ray continuum from $511 \mathrm{keV}$ downwards. In older magnetars with weaker MFs, this process will soon stop, while it will continue to softer photon energies in younger ones with stronger MFs, because this process, unlike the $\mathrm{e}^{+} \mathrm{e}^{-}$pair creation, has no particular energy threshold. This scenario, ${ }^{49)}$ fully based on the magnetar hypothesis, can consistently explain the properties of the hard component, including its evolution revealed with Suzaku.

The above hypothesis provides a nice research subject for future observations in the $0.1-1 \mathrm{MeV}$ range. If we can detect a steep cutoff of the continuum above $\sim 511 \mathrm{keV}$, our scenario, as well as the magnetar hypothesis itself, will be significantly reinforced.

4.3. Bursts and persistent signals. As explained in $\S 4.1$ (item 2), the X-ray/gamma-ray bursts emitted from an activated magnetar scatter over many orders of magnitude in their size (energy content). Among these bursts, very energetic and rare ones have been detected and studied by instruments with a wide sky coverage (but with a low sensitivity), because their occurrence is totally unpredictable. In contrast, little has been known about broad-band properties of smaller bursts that are undetectable by these wide-field instruments. We have successfully carried out this challenge with Suzaku, through Target of Opportunity observations (triggered by Swift) of two activated magnetars; the 
new magnetar SGR 0501+4516 ${ }^{50)}$ which suddenly appeared in 2008 August, and the fastest-spinning known magnetar 1E 1547.0-5408 ${ }^{51)}$ which became active in 2009 January.

Figure 8 shows the results on SGR 0501+ $4516,{ }^{50), 52), 53)}$ where we compare wide-band spectra of (i) one big burst (which caused an automatic shut-off of the HXD), (ii) stacked small bursts, (iii) persistent emission during the activity, and (iv) that after the activity decreased. Thus, the spectra of (ii) and (iii) both consist of the soft and hard components, and the upper limit in (iii) is also consistent. In (i), the two components appear to be merged together. Thus, the two-component nature of the magnetar emission has been found to be a basic property common to both their persistent and burst emissions. In addition, over more than 4 orders of magnitude, the luminosities of the two components are approximately proportional to each other. These results strongly suggest that the persistent and burst emissions are produced essentially by the same mechanism. We obtained similar results from $1 \mathrm{E}$ $1547.0-5408 .^{51), 54), 55)}$

Incorporating data from a small satellite HETE2, Nakagawa et al. ${ }^{56)}$ proposed that the persistent emission from magnetars is an assembly of numerous "micro bursts" that are individually too small to detect. (Evidently, this was inspired by the well known view that solar coronae can be composed of numerous micro flares.) This conjecture can be

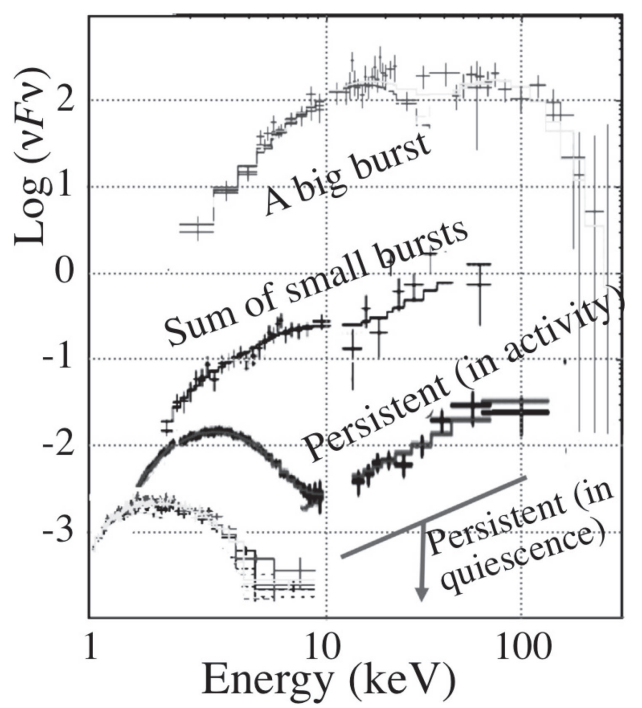

Fig. 8. Four spectra of the transient magnetar SGR $0501+4516$ obtained with Suzaku. A big burst, ${ }^{50)}$ a stack of small bursts, ${ }^{53}$ ) the persistent emission during the acivity, $\left.{ }^{52}\right)$ and that after the activity diminished. combined without difficulty with our new interpretation of the hard component (§ 4.2). In fact, individual bursts are likely to be a manifestation of sudden release of magnetic energies ${ }^{44)}$ somewhere in the system. This will lead to intense particle acceleration, and to the hard-tail formation via the proposed photon-splitting process. The scenario is hence attractive, but it requires that the number vs. size distribution of bursts should steepen considerably towards smaller bursts.

As already mentioned, the soft component of magnetars, with a luminosity comparable to that of the hard component, can be naturally regarded as thermal emission from the NS surface, heated either directly by the magnetic energy release, or indirectly by the accelerated particles. Actually, the softcomponent spectra can be approximated by a blackbody model, as inferred from Fig. $7 \mathrm{~b}$ and Fig. 8 . However, the data often require two temperatures, with the hotter one $\sim 3$ times higher than the other. Because this universal scaling applies to both persistent and burst spectra, ${ }^{56), 57)}$ the two-temperature property is considered to represent some physics specific to the strong MF. One possibility is that electrons under such strong MF have quite different scattering cross sections to the two X-ray polarizasion modes, $\mathrm{O}$-mode and $\mathrm{X}$-mode with respect to the $\mathrm{MF}$ direction. As a result, the two modes may have different photospheres with different temperatures. If this interpretation is correct, we expect the soft component to be strongly polarized. The verification of this idea must await the advent of X-ray polarimetry missions, including in particular PRAXYS being proposed under US-Japan collaboration.

4.4. Relation to supernova remnants. Usually, stars with the initial mass $>25 M_{\odot}$ are thought to leave black holes, rather than NSs, in their collapse $(\S 2)$. However, if the star is rapidly rotating, and/or is strongly magnetized, excess energies in the rotation and/or MF can expel out a larger amount of mass, and can lead to the formation of an NS rather than a black hole. It has hence been proposed that magnetars can be produced in supernova explosions of very massive (e.g., initial mass $>20 M_{\odot}$ ) and rapidlyrotating stars. ${ }^{58)}$

Using Suzaku, we examined the above prediction, since past observations were not very informative. For this purpose, we chose the SNR called CTB109, which hosts at its center a relatively aged magnetar, named 1E 2256+586. As shown in Fig. 9, this is actually one of the most typical magnetarSNR associations. By analyzing soft X-ray spectra 


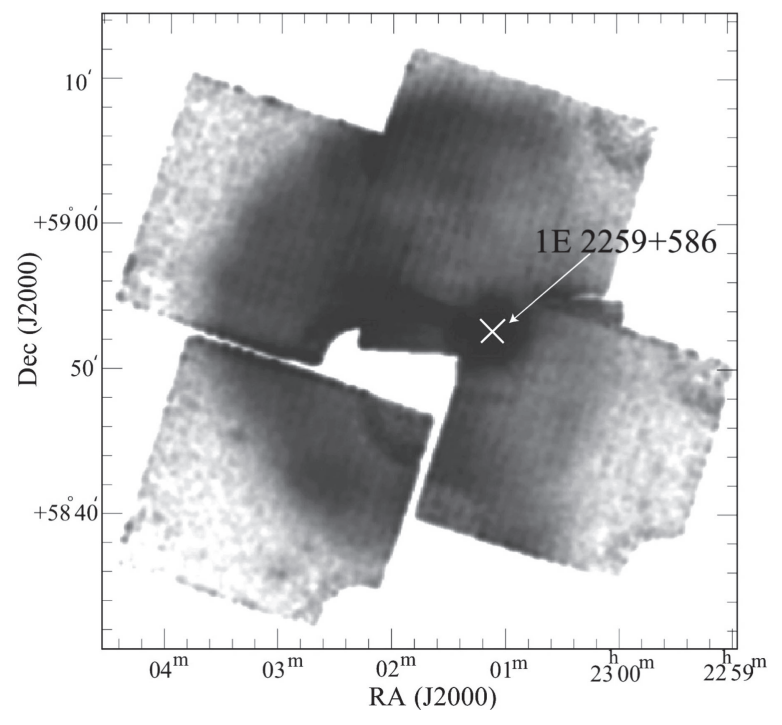

Fig. 9. A mosaic Suzaku image (0.5-4keV) of the SNR CTB109, hosting the magnetar $1 \mathrm{E} 2258+586$ at its center. ${ }^{60)}$

of CTB109 obtained with the Suzaku XIS, Nakano $(2015)^{59)}$ found that the X-ray emitting plasma involves two temperatures $\left(\sim 7 \times 10^{6} \mathrm{~K}\right.$ and $\sim 2 \times$ $\left.10^{6} \mathrm{~K}\right)$. He identified the hotter and cooler components to the stellar ejecta and shock-heated interstellar matter, respectively, and estimated the former to have a mass of $\sim 40 M_{\odot}$. This provides one of the first observational confirmations of the theoretical prediction described above.

As seen in Fig. 9, 1E 2259+586 resides at the center of the half-moon-shaped CTB109. Nevertheless, their mutual association was plagued with a serious puzzle called "age problem": the characteristic age of $1 \mathrm{E} 2259+586, \tau_{\mathrm{c}}=230 \mathrm{kyr}$, is much longer than the estimated age of CTB109, $13 \mathrm{kyr}$. This puzzle has been successfully solved ${ }^{60)}$ by considering that $\tau_{\mathrm{c}}$ calculated via Eq. [5] assuming a constant $B_{\mathrm{d}}$ systematically overestimates the true system age when the MF strength is decreasing with time as postulated in the magnetar hypothesis. Specifically, they mathematically modeled the spin down and the MF decay of $1 \mathrm{E} 2259+586$, and constructed the following scenario for this magnetar-SNR association. ${ }^{60)}$

1. CTB109 and 1E 2259+586 were born together, $\sim 13 \mathrm{kyr}$ (not $230 \mathrm{kyr}$ ) ago, by the supernova explosion of a progenitor of which the initial mass was $\sim 40 M_{\odot}$

2. At the birth, $1 \mathrm{E} 2259+586$ was rapidly rotating (e.g., $P \sim 10 \mathrm{msec}$ ), and had a higher $B_{\mathrm{d}}(e . g$. , $\left.\sim 10^{11} \mathrm{~T}\right)$ than the present value of $B_{\mathrm{d}}=5.9 \times$ $10^{9} \mathrm{~T}$.
3. Because of the strong initial MF, 1E $2259+586$ quickly [Eq. [20]] dumped out most of its rotational energy and became a slow rotator. Meantime, the MF decayed by dissipating $E_{\mathrm{mag}}$, to make $\dot{P}$ smaller and smaller.

4. As a result, $1 \mathrm{E} 2259+586$ has achieved the present values of $P=6.98 \mathrm{~s}$ and $\dot{P}=4.8 \times$ $10^{-13} \mathrm{~s} \mathrm{~s}^{-1}$ in a much shorter time, than $\tau_{\mathrm{c}}$ calculated backwards from the present-day values ignoring the MF decay.

The above scenario, derived from the particular SNR-magnetar pair, does not conflict with observed properties of other magnetar-SNR associations, and have three important and more general implications for magnetars. First, it implies that 1E 2259+586 (and possibly other magnetars as well) is indeed a magnetically-powered NS, because its MF must have been decreasing significantly as indicated by the age problem. Second, the scenario can also explain why the values of $P$ of magnetars are concentrated in the narrow range of $2-12 \mathrm{sec}$. Finally, magnetars are thus considered to be systematically and considerably younger than is indicated by their face values of $\tau_{\mathrm{c}}$. This last point is of the largest importance, because it further implies that magnetars must be produced with a much higher rate than thought previously, possibly more frequently than radio pulsars. The produced magnetars will fade off very quickly by exhausting $E_{\mathrm{mag}}$, and become invisible, because they can no longer be rotation-powered objects. These results altogether reinforce the magnetar hypothesis significantly, and suggest that magnetars are in fact one of major forms of new-born NSs, instead of being a rare and special population.

4.5. Free precession of magnetars. In addition to the dipole MFs which we have considered so far, magnetars can also harbor strong toroidal $\mathrm{MF}, B_{\mathrm{t}}$, which is confined inside the stars. In fact, a few magnetars which were discovered recently have $B_{\mathrm{d}}<B_{\mathrm{cr}}{ }^{61), 62)}$ so that the burst activity which led to their discovery is likely to be powered by much stronger $B_{\mathrm{t}}$ hidden inside them. Such toroidal MFs could be produced in the final collapse of a stellar core, wherein the field lines will be wound up by differential rotation. ${ }^{63)}$ Although it is intrinsically difficult to observationally estimate $B_{\mathrm{t}}$ which is invisible from outside, we have successfully overcome this difficulty, by utilizing the idea that strong internal MFs will deform the star up to an "asphericity" of ${ }^{64)}$

$$
\epsilon \equiv\left(I_{1}-I_{3}\right) / I_{3} \sim 1 \times 10^{-4}\left(B_{\mathrm{t}} / 10^{12} \mathrm{~T}\right) .
$$


Here, $I_{3}$ is the moment of inertia around the magnetar's symmetry axis $\hat{x}_{3}$, which we identify with its dipole MF axis, and $I_{1}$ is that around the axes orthogonal to $\hat{x}_{3}$. The deformation is expected to be prolate $(\varepsilon>0)$ if $B_{\mathrm{t}} \gg B_{\mathrm{d}}$ as assumed below, while oblate $(\varepsilon<0)$ if $B_{\mathrm{d}}$ dominates.

Let us consider dynamics of such an axisymmetric rigid body, under no external torque. If $\hat{x}_{3} \| \vec{L}$, where $\vec{L}$ is the angular momentum, the body would simply rotate around $\vec{L}$ with a constant rotation period $P_{\text {rot }}=2 \pi I_{3} / L$ (with $\left.L \equiv|\vec{L}|\right)$. However, if $\hat{x}_{3}$ is titled from $\vec{L}$ by a finite "wobbling angle" $\alpha$ (which can take any value regardless of $\epsilon$ or $|L|)$, the $\hat{x}_{3}$ axis will rotate, or wobble, around $\vec{L}$ with a slightly different "precession period" $P_{\text {prec }}=2 \pi I_{1} / L$. This condition is illustrated in Fig. 10. As seen from an observer located on the $\vec{L}-\hat{x}_{3}$ plane, the body will slowly rotates at a "slip period",

$$
T \equiv P_{\text {prec }} / \epsilon=\left(1 / P_{\text {rot }}-1 / P_{\text {prec }}\right)^{-1},
$$

which is just the beat between $P_{\text {prec }}$ and $P_{\text {rot }}$. This is the most basic behavior of a torque-free rigid body with axial symmetry, and is called "free precession". ${ }^{65)}$

Even when the free precession is taking place, (i.e., $\epsilon \neq 0$ and $\alpha \neq 0$ ), we can detect only $P_{\text {prec }}$, and cannot observe $P_{\text {rot }}$, as long as NS's radiation pattern is symmetric around $\hat{x}_{3}$. (In this sense, the regular pulsations of radio pulsars and BXPs should be considered as precession, rather than rotation.) However, if the radiation pattern is asymmetric around $\hat{x}_{3}$, it becomes possible to observe the slip period $T$ in addition to $P_{\text {prec }}$. Suppose that the emission hotspot (an orange circle in Fig. 10) is displaced from $\hat{x}_{3}$ by a finite angle $\gamma$, or the radiation beam is tilted by an angle $\xi$ from $\hat{x}_{3}$. Then, depending on the phase in $T$, the emission reaching us will

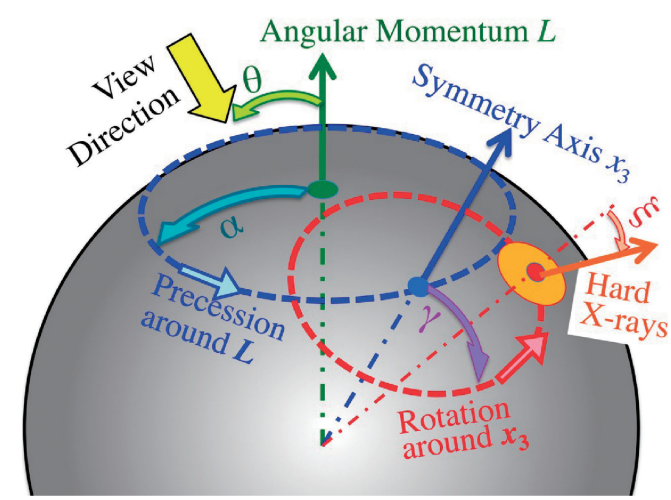

Fig. 10. An illustration of free precession of an axisymmetric rigid body. Three angles, $\alpha, \gamma$, and $\xi$ are of importance. See text for details. become maximum slightly before or after the timing when the $\vec{L}-\hat{x}_{3}$ plane points to us every precession cycle. ${ }^{65)}$ As a result, the regular pulsation at $P_{\text {prec }}$ becomes periodically phase modulated, at the period $T$ and with a certain modulation amplitude $A_{\mathrm{p}} \ll$ $P_{\text {prec }}$. This $A_{\mathrm{p}}$ is expected to depend positively on $\alpha$, $\gamma$, and $\xi$.

The persistently bright X-ray source $4 \mathrm{U}$ $0142+61$, pulsing at $P=8.689 \mathrm{sec}$, is one of the magnetars with the strongest hard component, showing a clearcut two-component spectrum (blue in Fig. 7b). ${ }^{66)}$ We analyzed 4 Suzaku data sets of this object, taken in 2007, 2009, 2011, and 2013. Clear evidence of periodic modulation in the $8.689 \mathrm{sec}$ pulse phase was discovered in the $15-40 \mathrm{keV}$ data from the latter 3 observations, at a consistent modulation period (to be identified with the slip period) of $T=$ $55 \pm 4$ ksec. $^{67)}$ The modulation amplitude in 2009, 2011 , and 2013 was $A_{\mathrm{p}}=0.7 \pm 0.3 \mathrm{sec}, 0.9 \pm 0.5 \mathrm{sec}$, and $1.1 \pm 0.4 \mathrm{sec}$, respectively, while the 2007 data gave an upper limit as $A_{\mathrm{p}}<0.8$. Interestingly, the phase modulation was always absent $\left(A_{\mathrm{p}}<0.3 \mathrm{sec}\right)$ in signals below $10 \mathrm{keV}$, where the soft component dominates (Fig. 7b). These novel results on $4 \mathrm{U}$ $0142+61$ can be consistently interpreted by assuming that this magnetar is axisymmetric, and is undergoing free precession with a slip period of $T=55 \mathrm{ksec}$. From Eq. [31], the asphericity is constrained as $\epsilon=$ $1.6 \times 10^{-4}$, which in turn translates to $B_{\mathrm{t}} \sim 10^{12} \mathrm{~T}$ via Eq. [30] if the deformation is attributed to internal magnetic pressure. The absence of this phase modulation in the soft component can be understood if the soft $\mathrm{X}$-ray emission region is symmetric $\left(\gamma=\xi=0\right.$ in Fig. 10) around $\hat{x}_{3}$, while the possible year-to-year variation in $A_{\mathrm{p}}$ in the hard component is likely to result from secular changes in $\gamma(\neq 0)$ and/or $\xi(\neq 0)$ of the hard X-ray emission region; $\alpha$ should not change on such short time scales. The data however constrained neither $\alpha, \gamma$, nor $\xi$ uniquely.

Although we cannot tell from the data alone whether the suggested deformation of $4 \mathrm{U} 0142+61$ is prolate or oblate, we may obtain a clue to this issue from basic Newtonian dynamics. When $\vec{L}$ is conserved and some energy dissipation takes place, an oblate rigid body will reach its energy minimum at $\alpha=0$ (no precession), while a prolate body will do so at $\alpha=90^{\circ}$ (flat spin). Since internal dissipation in an NS is considered to be relatively fast, the wobbling angle of an oblate NS (e.g., due to centrifugal force when the rotation is fast) would soon decay even if free precession is once excited by some perturbation. Since $4 \mathrm{U} 0142+61$ definitely has $\alpha \neq 0$ as evidence 
by its clear pulsation, a prolate shape is favored, in which case $\alpha$ would gradually increase with time.

The same pulse-phase modulation effects, as observed from $4 \mathrm{U} 0142+61$, have also been discovered in Suzaku data of 1E 1547.0-4516, ${ }^{68)}$ the fastestspinning $(P=2.0721 \mathrm{sec})$ magnetar which became active in 2009 January ( $\S 4.3$; orange in Fig. $7 b) .{ }^{51)}$ In this case, we obtained $T=36.0_{-2.5}^{+4.5} \mathrm{ksec}$, which yields $\epsilon=0.6 \times 10^{-4}$. This is somewhat smaller than, but still of the same order, as the case of $4 \mathrm{U} 0142+61$. Figure 11 visualizes the phase modulation of the 15$40 \mathrm{keV}$ pulse profiles of this magnetar at the $36 \mathrm{ksec}$ slip period. Again, the pulse-phase modulation was seen only in the hard component, and absent in the soft component.

The study of free precession has opened a totally new window on the estimation of toroidal MFs of magnetars. We are now searching Suzaku data of other magnetars for similar effects. In addition, archival data from the $N u S T A R$ mission will greatly accelerate the study. An increased number of detections of the free precession will allow us to address such issues as; whether the magnetic deformation is common among magnetars; what is the distribution of $B_{\mathrm{t}}$; and whether $B_{\mathrm{t}}$ is proportional to $B_{\mathrm{t}}$.

\section{Discussion}

As described so far, our understanding of the NS magnetism has made a large progress owing to the extensive studies over the last quarter century. Nevertheless, we are still left with several fundamental questions to be solved, as we already mentioned in $\S 2.3, \S 3.4$, and several places in $\S 4$. These include; why the MF of NSs exhibits such a large scatter as seen in Fig. 4 even though their masses and radii show narrow distributions; are there intermediate objects between magnetars and ordinary radio pulsars; how the MF of NSs evolve after their birth; and, what hold the MFs inside NSs. In an attempt to answer some of these questions, let us conduct discussion from several aspects.

5.1. Initial MFs of NSs. In the last decade, significant new facts about magnetars have been unveiled (§ 4). These include our own achievements; the universal two-component nature of their persistent and short-burst spectra ( $\$ 4.2)$; the new scaling law for the hard component $(\S 4.2)$; the microburst hypothesis to explain the persistent emission $(\S 4.3)$; the two-temperature quantification of their soft component $(\S 4.3)$; the estimated progenitor mass of $1 \mathrm{E} 2259+586(\S 4.4)$; the solution to the age problem in terms of MF decay $(\S 4.4)$; and the evidence for free precession in the two magnetars $(\S 4.5)$. As a result, our confidence in the magnetar hypothesis has been significantly reinforced, and evidence is accumulating to believe that magnetars really have strong MFs as $B_{\mathrm{d}}=10^{10}-10^{11} \mathrm{~T}$ or higher, and are powered by the magnetic energies. Furthermore, we have shown that magnetars can be one of the most major subclasses of new-born NSs, besides radio pulsars and BXPs. As a natural consequence of these results, NSs are now considered to have a wide range of scatter in $B_{\mathrm{d}}$ from their birth, at least over $10^{8-11} \mathrm{~T}$.

Can a new-born NS, then, have a very weak MF as $B_{\mathrm{d}}<10^{7} \mathrm{~T}$ ? The answer is probably yes, from the following two pieces of evidence. One is a handful young SNRs (with age $<10 \mathrm{kyr}$; including Cas A in particular) that contain rather inactive central NSs called CCOs (Central Compact Objects). Some of CCOs are weakly pulsing at sub-second periods, and their values of $\dot{P}$ is extremely small, implying $B_{\mathrm{d}} \sin \theta=10^{6}-10^{7} \mathrm{~T}$. Even if $\sin \theta \sim 0$, we would expect strong particle acceleration and associated activities as long as $B_{\mathrm{d}} \sim 10^{8} \mathrm{~T} .{ }^{10)}$ Thus, CCOs are considered to have truly weak $B_{\mathrm{d}}$. The other is a peculiar accreting NS called Cir X-1. It has a rather massive $\left(\sim 10 M_{\odot}\right)$ companion, of which the lifetime is $<10^{7.5}$ year, and could even be associated with the SNR G321.9-0.3. Therefore, this NS is definitely very young, but it it known to emit Type I X-ray bursts, which are considered to take place only if $B_{\mathrm{d}}<10^{6} \mathrm{~T}$ or so. Therefore, this particular NS is also considered young and weakly magnetized. These two examples suggest that some NSs are born with weak dipole MFs (although they could have high $B_{\mathrm{t}}$ ).

Based on these considerations, we conclude that NSs are born with a variety of dipole MF strengths, ranging from $>10^{11} \mathrm{~T}$ down to $B_{\mathrm{d}}<10^{7}$. Objects like the Crab Pulsar can no longer be considered as typical young NSs. The information on $B_{\mathrm{t}}$ is still very poor, however.

5.2. Evolution of MFs above $\boldsymbol{B}_{\mathrm{cr}}$. When trying to understand the MF distribution in Fig. 6, three factors must be considered; the initial $\mathrm{MF}$ spread as discussed in $\S 5.1$, the MF evolution over the life times of respective NSs, and various observational selection effects. Here, let us consider the second factor, incorporating some aspects of the third one. For this purpose, Fig. 12 summarizes all the currently known non-accreting pulsars (including magnetars) on the plane of $\tau_{\mathrm{c}}$ and $B_{\mathrm{d}}$. Several remarks may be added to this figure. (i) As long as non-accreting pulsars are concerned, this plot can be 


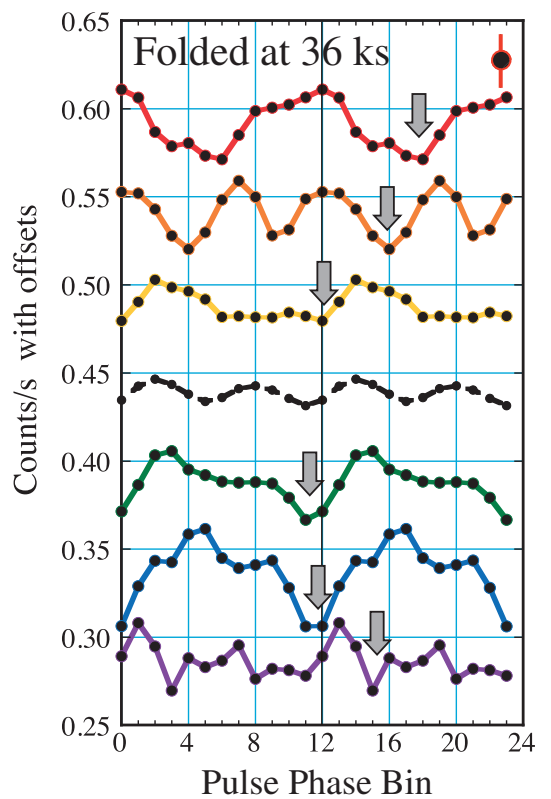

Fig. 11. The 15-40 keV pulse profiles of the magnetar $1 \mathrm{E}$ 1547.0-4516, folded at a barycentric period of $2.07214 \mathrm{sec}$, in 6 different phases of the $T=36 \mathrm{ksec}$ slip period. ${ }^{68)}$ Their average is shown in black at the middle.

obtained by changing the plotting axes in Fig. 4, and its projection onto the $B_{\mathrm{d}}$ axis gives Fig. 6 (except the green histogram). (ii) The green squares indicate pulsars in binaries, but they have relatively wide orbits so that mass accretion does not take place. (iii) The values of $\tau_{\mathrm{c}}$ of magnetars are likely to be systematically overestimated as we described in $\S 4.4$, but the plot employs the uncorrected $\tau_{\mathrm{c}}$. (iv) A few SNR-hosted pulsars with $\tau_{\mathrm{c}}=10^{6}-10^{9} \mathrm{yr}$ and $B_{\mathrm{d}}=$ $10^{5}-10^{6} \mathrm{~T}$ are CCOs, of which $\tau_{\mathrm{c}}$ is likely to be heavily overestimated, because we cannot ignore in this case $t_{1}$ in Eq. [18]. With these remarks in mind, let us investigate into Fig. 12.

In Fig. 12, magnetars define a clear negative dependence of $B_{\mathrm{d}}$ on $\tau_{\mathrm{c}}$. This behavior is likely to be free from selection effects, since persistent magnetars and newly discovered transients line up on the same line. The trend agrees with the magnetar hypothesis, and our results on 1E 2259+586 and CTB109 (§ 4.4). More quantitatively, we can model the decay of $B_{\mathrm{d}}$ of magnetars as ${ }^{60), 69)}$

$$
B_{\mathrm{d}}(T)=B_{0}\left\{\left(1+\frac{\beta t}{\tau_{\mathrm{d}}}\right)\right\}^{-1 / \beta},
$$

where $t$ is the time since the birth, $B_{0}$ is the initial $(t=0)$ field, $\beta$ is a positive parameter, and $\tau_{\mathrm{d}} / \beta$ describes a time scale on which the initial $B_{0}$ is

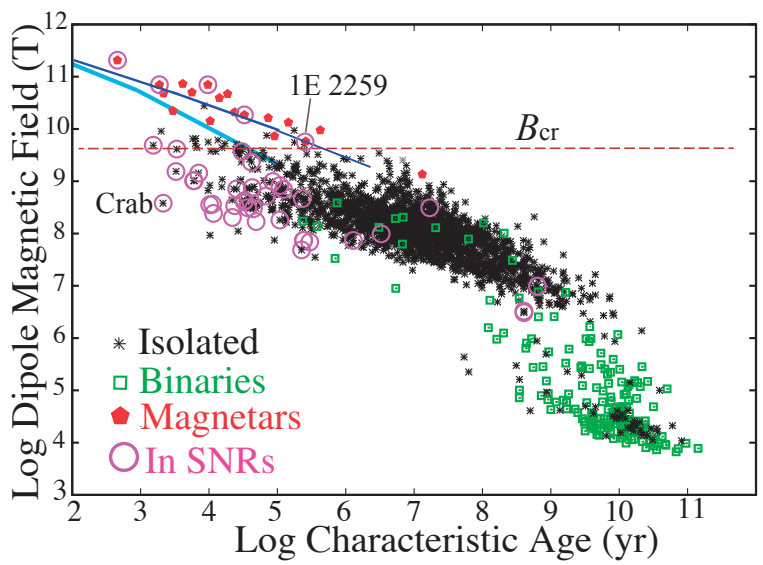

Fig. 12. The dipole magnetic fields and the characteristic ages of all known pulsars, including magnetars but excluding massaccreting objects such as BXPs and LMXBs. Black crosses, green squares, and red hexagons indicate isolated pulsars, pulsars in binaries, and magnetars, respectively. Magenta circles indicate those in SNRs. The plot was made by T. Nakano, making use of the information from the ATNF Pulsar Catalogue ${ }^{76)}$ in the same way as Fig. 6. Cyan and blue lines indicate possible evolutionary tracks of $1 \mathrm{E} 2259+586$ (see text $\S 5.1$ ).

halved. The condition of a constant MF (non-decay) is expressed as $\tau_{\mathrm{d}} \rightarrow \infty$.

In the case of $1 \mathrm{E} 2259+586$, one possible solution (among many possibilities) to Eq. [32] is given as $B_{0}=1.8 \times 10^{11} \mathrm{~T}, \tau_{\mathrm{d}}=160 \mathrm{yr}$, and $\beta=1.4,{ }^{60)}$ which imply that the MF decreased to $0.033 B_{0}$ in the $13 \mathrm{ky}$ of life time. This particular evolutionary track is shown in Fig. 12 by a cyan curve, in which abscissa is redefined as the true elapsed time. After converting this true age to the apparent (heavily over-estimated) $\tau_{\mathrm{c}}$ to match with the plot, the track becomes as indicated by a blue curve. Thus, Eq. [32] can explain the behavior of not only $1 \mathrm{E} 2259+586$, but also of other magnetars. Furthermore, the magnetic energy release rate, $-d E_{\mathrm{mag}} / d t$, calculated from Eq. [12] and Eq. [32] using these parameters, become $\sim 7.5 \times$ $10^{27} \mathrm{~W}$ at present, which is in a good agreement with the observed bolometric X-ray luminosity of $1 \mathrm{E}$ $2259+586,6 \times 10^{27} \mathrm{~W}$. From these considerations, the MFs of magnetars are concluded to decay over time scales of 10-100 kyr, down to values below $B_{\text {cr. }}$. They would soon become undetectable, unless some revival mechanisms operate.

5.3. Evolution of MFs below $\boldsymbol{B}_{\text {cr. }}$. Hereafter, we limit the discussion to those NS which were born with $B_{0}<B_{\text {cr. }}$. In Fig. 12, radio pulsars are distributed approximately along a slope of $B_{\mathrm{d}} \propto \tau_{\mathrm{c}}^{-0.4}$. The simplest interpretation, which in fact used to be a standard one till the 1990 's, is to consider that the 
MF of NSs decay gradually and ubiquitously, on typical e-folding time scales of $\sim 10^{8} \mathrm{yr}^{70}{ }^{7}$ This was also motivated to explain MSPs and LMXBs: a standard view is that they were born as isolated radio pulsars with $B_{\mathrm{d}} \sim 10^{8} \mathrm{~T}$, became slow rotators by losing $E_{\text {rot }}$, and weak- $B_{\mathrm{d}}$ objects due to the MF decay, and captured by a low-mass star to become LMXBs, wherein they were spun up ("recycled") by accretion as described by Eq. [14]. After the accretion ceased or the binary dissociated, the NS were left as MSPs. ${ }^{71}$ )

The above scenario of MF decay, however, became obsolete for the following two reasons. One is selection effects, combined with the initial scatter in $B_{0}(\S 5.1)$. Pulsars born with relatively high $B_{0}$, hence with shorter lifetimes [Eq. [20]], will fade away more quickly than those with weaker $B_{0}$. Furthermore, those with weak $B_{0}$ have large values of $t_{1}$ so that their $\tau_{\mathrm{c}}$ will be overestimated. These two artifacts suppress, in Fig. 12, the numbers of old/ high- $B_{\mathrm{d}}$ pulsars and young/low- $B_{\mathrm{d}}$ ones, respectively, to mimic the apparent negative correlation. Actually, a reanalysis of the radio pulsar statistics had already shown in the middle 1990's that a constant MF model is consistent with the observations. ${ }^{72}$ )

The other evidence is the very narrow distribution of $B_{\mathrm{d}}$ of BXPs [Fig. 6 (green) and Eq. [29]], which is considered complete on the lower-field side $^{17), 18)}$ (although still incomplete towards the higher-field side). Considering typical lifetimes of the primary stars of BXPs $\left(10^{5.5}-10^{7.5} \mathrm{yr}\right)$, any field decay on time scales of $<10^{8}$ yr would make the MF distribution of BXPs much broader towards the lower field side. Furthermore, the presence of the three BXPs with low-mass companions stars (§ 3.3), which have estimated system ages of $>10^{9} \mathrm{yr}$, cannot be reconciled with the MF decay even on a time scale of $10^{9} \mathrm{yr}$. Therefore, the MF below $B_{\mathrm{d}} \sim 10^{8} \mathrm{~T}$ is unlikely to decay smoothly on time scales of $<10^{9} \mathrm{yr}$, although the behavior at $10^{8} \mathrm{~T}<B_{\mathrm{d}}<B_{\text {cr }}$ is still uncertain.

An alternative view to the above MF decay scenario is to assume that the MF of NSs remain relatively constant except in magnetars, and the scatter in Fig. 6 and Fig. 12 below $B_{\mathrm{d}} \sim 10^{9} \mathrm{~T}$ is at least partially natal, i.e., determined by distributions in $B_{0}$. Then, can we explain the larger populations of non-accreting binary MSPs (green squares in Fig. 12) and accreting LMXBs ( 200 known)? These aged NSs in binaries are likely to have been produced via capture of NSs by non-degenerate stars, or via the accretion-induced collapse ${ }^{7)}$ of white dwarfs, because they all have low-mass companions: it is unlikely that such a low-mass star formed a binary with a massive progenitor of the NS. However, if the capture process dominates, we would observe even larger number of strong-MF NS with low-mass companions (in contrast to the only known three), because higher MFs of NSs will enhance the extraction of orbital angular momentum ${ }^{73)}$ which is necessary in their capture process. Even if the accretion induced collapse dominates, a small fraction (10-25\%) of white dwarfs with $B_{\mathrm{d}}>10^{2} \mathrm{~T}$ would become NSs with $B_{\mathrm{d}}>10^{8} \mathrm{~T}$ via the magnetic flux compression; again, we should observe more aged NSs with strong MFs than are observed. Therefore, the MF below $\sim 10^{9}$ is unlikely to be constant with time.

A third explanation of the MF evolution below $B_{\mathrm{d}} \sim 10^{9} \mathrm{~T}$ is to invoke a kind of "transition" from $B_{\mathrm{d}}=10^{7}-10^{9} \mathrm{~T}$ to $10^{4}-10^{5} \mathrm{~T}$, at a certain timing of the evolution of individual NSs. As a toy model, we may assume that the transition occurs in $\sim 10^{9} \mathrm{yr}$, and that the timing scatters considerably from object to object for some unspecified reasons. This is indeed what is apparently suggested by Fig. 12. Then, the strong concentration of the MF of BXPs at Eq. [29] can be explained because their life times are $<10^{8} \mathrm{yr}$ or so. The abundance of MSPs and LMXBs can be explained by considering that the dominant NS subclass born with $B_{\mathrm{d}}=10^{7}-10^{9} \mathrm{~T}$ would have mostly completed their transition in $\sim 10^{9} \mathrm{yr}$. The three outliers, Her X-1, 4U 1626-67, and 4U 182237 , can be interpreted as those which have not yet completed their transition. Thus, this simple view $^{17), 18)}$ can explain the observations in a consistent way, although it is not yet widely accepted.

5.4. How the MF is held. The final and the most fundamental issue is how the MFs of NSs are held, and how the strong-to-weak MF transition suggested in $\S 5.3$ takes place. The simplest idea is so-called fossil-MF view; during the core collapse, the progenitor's MF was compressed $(\S 2.2)$ to $\sim 10^{8} \mathrm{~T}$, and is sustained presumably by toroidal electric current, carried by protons and/or electrons that remain in the NS interior (mainly in so-called inner crust). The protons are possibly in a superfluid state, and are considered to gyrate around digitized "vortices" which thread the superfluid. ${ }^{74}$ These current-carrying vortices are considered to be gradually transported outwards, leading to the MF decay. However, as argued in $\S 5.3$, this classical view can no longer explain the MF evolution. Furthermore, the ultra-strong MFs of magnetars may not be explained by such a fossil view. We are hence urged to explore alternative explanations. 
A contrasting scenario is to assume that the $\mathrm{MF}$ is sustained by ferromagnetism in nuclear matter, produced by quantum alignment of magnetic moment of neutrons. ${ }^{17), 18)}$ This view had already been proposed in the late 1960 's,${ }^{75)}$ immediately after the discovery of pulsars. ${ }^{11)}$ If a fraction $f$ of the total $N_{\mathrm{n}}=M_{\mathrm{NS}} / m_{\mathrm{n}}=1.7 \times 10^{57}$ neutrons in an NS become spin-aligned, we obtain from Eq. [4] a MF strength of

$$
B \sim \mu_{0} \mu_{\mathrm{n}} f N_{\mathrm{n}} / 2 \pi R_{\mathrm{NS}}^{3} \sim 2.5 f \times 10^{12} \mathrm{~T}
$$

where $\mu_{\mathrm{n}}=1.91 \mu_{\mathrm{N}}=9.7 \times 10^{-27} \mathrm{~J} \mathrm{~T}^{-1}$ is the neutron magnetic moment, with $\mu_{\mathrm{N}} \equiv e \hbar / 2 m_{\mathrm{p}}=5.05 \times$ $10^{-27} \mathrm{~J} \mathrm{~T}^{-1}$ the nuclear magneton and 1.91 being the neutron's $g$-factor halved. Thus, as illustrated in Fig. 2, we can explain the strongest MFs of magnetars if $f \sim 1$, and those of ordinary pulsars if only $0.1 \%$ of the neutrons form a ferromagnetic phase. The MF transition postulated in $\S 5.3$ can be readily explained as ferromagnetic to paramagnetic state transitions of the nuclear matter, in response to, e.g., slight changes in the internal temperature, possibly during high-accretion-rate phase as LMXBs. However, a weak point of this idea is that quantum exchange force between two neutrons prefers singlet$\mathrm{S}$ state, wherein the wave function is symmetric against positional exchange of the two neutrons and anti-symmetric with respect to their spin factor, than triplet-P state wherein the two spins become aligned.

Yet another possibility is that the electrons remaining in the NS becomes spin-aligned to form a ferromagnetic phase, ${ }^{77)}$ due to some quantum mechanisms, including the neutrino's chirality violation, ${ }^{78)}$ or "chiral plasma instability" through which toroidal and poloidal electron currents are successively amplified. ${ }^{79)}$ Thus, MFs close to Eq. [33] could be produced, because the very small number (e.g., $\sim 10^{-3}$ of neutrons) of the remaining electrons can be compensated by the Bohr magneton, $\mu=e \hbar / 2 m_{\mathrm{e}}$, which is $\sim 1840$ times larger than $\mu_{\mathrm{N}}$. These new ideas are attractive, and should be extensively pursued, because they try to explain the NS magnetism in terms of symmetry in fundamental physics, including the electron-to-proton mass difference, the chirality violation by neutrinos, and the overall parity and charge asymmetry.

\section{Conclusion}

Over the past quarter century, we have been using several (mainly Japanese) X-ray observatories towards understanding the NS magnetism in the context of both astrophysics (formation and evolu- tion of NSs) and fundamental physics. The results obtained in these studies can be summarized into the following points.

1. Magnetars are indeed likely to be magneticallypowered objects, with truly super-critical dipole fields of $B_{\mathrm{d}}=10^{10}-10^{11} \mathrm{~T}$ and possibly even stronger toroidal fields of $B_{\mathrm{t}} \sim 10^{12} \mathrm{~T}$.

2. NSs are born with a wide range of initial dipole fields, from $10^{11}$ to $<10^{5} \mathrm{~T}$. In addition, magnetars are likely to occupy a relatively large fraction of NSs that are born in core-collapse supernovae.

3. The MFs of magnetars $\left(>B_{\text {cr }}\right)$ decay, in 10$100 \mathrm{kyr}$, as postulated in the original magnetar hypothesis.

4. The CRSF technique has provided a very important probe to the MF strengths of BXPs, and has reinforced the view that the $\mathrm{MF}$ is unlikely to make gradual decays except at $>B_{\mathrm{cr}}$.

5. The overall dipole-MF distributions of NSs, below $B_{\text {cr }}$, can be understood as combination of the initial MF scatter (item 2) and some kind of transition from strong-MF to weak-MF states.

6. The MF of NSs could be a manifestation of ferromagnetism in the nuclear matter, or more interestingly, a result of some symmetry braking in fundamental physics.

\section{Acknowledgements}

The author would like to thank Prof. Yasuo Tanaka, Prof. Naoki Onishi, Prof. Toshikazu Shigeyama, Dr. Tatehiro Mihara, Dr. Teruaki Enoto, and Dr. Toshio Nakano, for their inspiring discussion. His thanks are also due to his colleagues at the University of Tokyo and RIKEN. This work was supported by the MEXT KAKENHI on Innovative Areas, Area No. 2404, Grant No. 25105507, and MEXT KAKENHI Grant No. 15H03653.

\section{References}

1) Makishima, K. (2013) From Sco X-1 to magnetars: Past, present, and future of X-ray studies of neutron stars. Mem. Soc. Astron. Ital. 84, 547553.

2) Turner, M.J.L. et al. (1989) The large area counter on Ginga. Publ. Astron. Soc. Jpn. 41, 345-372.

3) Tanaka, Y., Inoue, H. and Holt, S.S. (1994) The X-ray astronomy satellite ASCA. Publ. Astron. Soc. Jpn. 46, L37-L41.

4) Mitsuda, K. et al. (2007) The X-ray observatory Suzaku. Publ. Astron. Soc. Jpn. 59, 1-7.

5) Matsuoka, M. et al. (2009) The MAXI mission on the 
ISS: Science and instruments for Monitoring Allsky X-ray Images. Publ. Astron. Soc. Jpn. 61, 9991010.

6) Apparao, K.M.V. (1973) The Crab Nebula. Astrophys. Space Sci. 25, 3.

7) Nomoto, K. and Kondo, Y. (1991) Conditions for accretion-induced collapse of white dwarfs. Astrophys. J. Lett. 367, L19-L22.

8) Özel, F., Psaltis, D., Narayan, R. and Santos Villarreal, A. (2012) On the mass distribution and birth masses of neutron stars. Astrophys. J. $\mathbf{7 5 7}$, id.55.

9) Ostriker, J. and Gunn, J.E. (1969) On the nature of pulsars. I. Theory. Astrophys. J. 157, 1395-1418.

10) Goldreich, P. and Julian, W.H. (1969) Pulsar electrodynamics. Astrophys. J. 157, 869-880.

11) Hewish, A., Bell, S.J., Pilkington, J.D.H., Scott, P.F. and Collins, R.A. (1968) Observation of a rapidly pulsating radio source. Nature 217, 709-713.

12) Abdo, A.A. et al. (2009) A population of gamma-ray emitting globular clusters seen with the Fermi Large Area Telescope. Science 325 (5942), 848851.

13) Lattimer, J.M. and Schutz, B.F. (2005) Constraining the equation of state with moment of inertia measurements. Astrophys. J. 629, 979-984.

14) Ghosh, P. and Lamb, F.K. (1979) Accretion by rotating magnetic neutron stars. III - Accretion torques and period changes in pulsating X-ray sources. Astrophys. J. 234, 296-316.

15) Sugizaki, M., Yamamoto, T., Mihara, T., Nakajima, M. and Makishima, K. (2015) Luminosity and spin-period evolution of GX 304-1 during outbursts from 2009 to 2013 observed with the MAXI/GSC, RXTE/PCA, and Fermi/GBM. Publ. Astron. Soc. Jpn. 67, id.73.

16) Takagi, T., Mihara, T., Sugizaki, M., Makishima, K. and Morii, M. (2016) Application of the Ghosh \& Lamb relation to the spin-up/down behavior in the X-ray binary pulsar 4U 1626-67. Publ. Astron. Soc. Jpn. 68 (in press).

17) Makishima, K., Mihara, T., Nagase, F. and Tanaka, Y. (1999) Cyclotron resonance effects in two binary X-ray pulsars and the evolution of neutron star magnetic fields. Astrophys. J. 525, 978-994.

18) Makishima, K. (2003) Measuring magnetic fields of neutron stars. Prog. Theor. Phys. 151, 54-63.

19) Trümper, J., Pietsch, W., Reppin, C., Voges, W., Staubert, R. and Kendziorra, E. (1978) Evidence for strong cyclotron line emission in the hard X-ray spectrum of Hercules X-1. Astrophys. J. 219, L105-L110.

20) Wheaton, W.A., Doty, J.P., Primini, F.A., Cooke, B.A., Dobson, C.A., Goldman, A., Hecht, M., Howe, S.K., Hoffman, J.A. and Scheepmaker, A. (1979) An absorption feature in the spectrum of the pulsed hard X-ray flux from $4 \mathrm{U} 0115+63$. Nature 282, 240-243.

21) Clark, G.W., Woo, J., Nagase, F., Makishima, K. and Sakao, T. (1990) Discovery of a cyclotron absorption line in the spectrum of the binary X-ray pulsar 4U 1538-52 observed by Ginga. Astrophys.
J. 353, 274-280.

22) Makishima, K. et al. (1990) Discovery of a prominent cyclotron absorption feature from the transient X-ray pulsar X0331+53. Astrophys. J. 365, L59L62.

23) Mihara, T., Makishima, K., Kamijo, S., Ohashi, T., Nagase, F., Tanaka, Y. and Koyama, K. (1991) Discovery of a cyclotron resonance feature at $30 \mathrm{keV}$ from the transient X-ray pulsar Cepheus X-4. Astrophys. J. Lett. 379, L61-L64.

24) Mihara, T., Makishima, K., Ohashi, T., Sakao, T. and Tashiro, M. (1990) New observations of the cyclotron absorption feature in Hercules X-1. Nature 346, 250-252.

25) Nagase, F., Dotani, T., Tanaka, Y., Makishima, K., Mihara, T., Sakao, T., Tsunemi, H., Kitamoto, S., Tamura, K., Yoshida, A. and Nakamura, H. (1991) Cyclotron line features in the spectrum of the transient X-ray pulsar X0115+634. Astrophys. J. Lett. 375, L49-L52.

26) Mihara, T. (1995) Observational study of X-ray spectra of binary pulsars with Ginga. PhD Thesis, The University of Tokyo.

27) Enoto, T. et al. (2008) Suzaku observations of Hercules X-1: Measurements of the two cyclotron harmonics. Publ. Astron. Soc. Jpn. 60, S57-S68.

28) dal Fiume, D., Orlandini, M., Frontera, F., del Sordo, S., Piraino, S., Santangelo, A., Segreto, A., Oosterbroek, T. and Parmar, A.N. (1999) The broad band spectral properties of galactic X-ray binary pulsars. Nucl. Phys. B Proc. Suppl. 69, $145-150$

29) Coburn, W., Heindl, W.A., Rothschild, R.E., Gruber, D.E., Kreykenbohm, I., Wilms, J., Kretschmar, P. and Staubert, R. (2002) Magnetic fields of accreting X-ray pulsars with the Rossi Xray timing explorer. Astrophys. J. 580, 394-412.

30) Santangelo, A. et al. (1999) A BeppoSAX study of the pulsating transient X0115+63: The first X-ray spectrum with four cyclotron harmonic features. Astrophys. J. Lett. 523, L85-L88.

31) Mihara, T., Makishima, K. and Nagase, F. (2004) Luminosity-related changes in the cyclotron resonance structure of the binary X-ray pulsar $4 \mathrm{U}$ 0115+63. Astrophys. J. 610, 390-401.

32) Nakajima, M., Mihara, T., Makishima, K. and Niko, H. (2006) A further study of the luminositydependent cyclotron resonance energies of the binary X-ray pulsar $4 \mathrm{U} 0115+63$ with the Rossi X-ray timing explorer. Astrophys. J. 646, 11251138.

33) Nakajima, M., Mihara, T. and Makishima, K. (2010) Energy-dependent harmonic ratios of the cyclotron features of X0331+53 in the 2004-2005 outburst. Astrophys. J. 710, 1755-1768.

34) Takahashi, T. et al. (2007) Hard X-ray Detector (HXD) on board Suzaku. Publ. Astron. Soc. Jpn. 59, S35-S51.

35) Terada, Y. et al. (2006) Cyclotron resonance energies at a low X-ray luminosity: A0535+262 observed with Suzaku. Astrophys. J. Lett. 648, L139-L142.

36) Yamamoto, T., Sugizaki, M., Mihara, T., Nakajima, 
M., Yamaoka, K., Matsuoka, M., Morii, M. and Makishima, K. (2011) Discovery of a cyclotron resonance feature in the X-ray spectrum of GX 304-1 with RXTE and Suzaku during outbursts detected by MAXI in 2010. Publ. Astron. Soc. Jpn. 63, S751-S757.

37) Suchy, S. et al. (2011) Suzaku observations of the HMXB 1A 1118-61. Astrophys. J. 733, id.15.

38) Yamamoto, T., Mihara, Ta., Sugizaki, M., Nakajima, M., Makishima, K. and Sasano, M. (2014) Firm detection of a cyclotron resonance feature with Suzaku in the X-ray spectrum of GRO J1008-57 during a giant outburst in 2012. Publ. Astron. Soc. Jpn. 66, id.59.

39) Iwakiri, W.B., Terada, Y., Mihara, T., Angelini, L., Tashiro, M.S., Enoto, T., Yamada, S., Makishima, K., Nakajima, M. and Yoshida, A. (2012) Possible detection of an emission cyclotron resonance scattering feature from the accretion-powered pulsar 4U 1626-67. Astrophys. J. 751, id.35.

40) Sasano, M., Makishima, K., Sakurai, S., Zhang, Z. and Enoto, T. (2014) Suzaku view of the neutron star in the dipping source $4 \mathrm{U}$ 1822-37. Publ. Astron. Soc. Jpn. 66, id.35.

41) Sasano, M. (2015) X-ray studies of highly magnetized neutron stars in binary systems with Suzaku. Ph.D. Thesis, The University of Tokyo.

42) Murakami, T., Tanaka, Y., Kulkarni, S.R., Ogasaka, Y., Sonobe, T., Ogawara, Y., Aoki, T. and Yoshida, A. (1994) X-ray identification of the soft $\gamma$-ray repeater 1806-20. Nature 368, 127-129.

43) Thompson, C. and Duncan, R.C. (1995) The soft gamma repeaters as very strongly magnetized neutron stars - I. Radiative mechanism for outbursts. Mon. Not. R. Astron. Soc. 275, 255-300.

44) Mereghetti, S. (2008) The strongest cosmic magnets: soft gamma-ray repeaters and anomalous X-ray pulsars. Astr. Ap. Review 15, 225-287.

45) Trümper, J., Dennerl, K., Kylafis, N. D., Ertan, Ü. and Zezas, A. (2013) An accretion model for the anomalous X-ray pulsar 4U $0142+61$. Astrophys. J. 764, id.49.

46) Kuiper, L., Hermsen, W., den Hartog, P.R. and Collmar, W. (2006) Discovery of luminous pulsed hard X-ray emission from anomalous X-ray pulsars 1RXS J1708-4009, 4U 0142+61, and 1E 2259+586 by INTEGRAL and RXTE. Astrophys. J. 645, 556-575.

47) den Hartog, P.R., Hermsen, W., Kuiper, L., Vink, J., in't Zand, J.J.M. and Collmar, W. (2006) INTEGRAL survey of the Cassiopeia region in hard X rays. Astron. Astrophys. 451, 587-602.

48) Pavlov, G.G., Zavlin, V.E., Sanwal, D., Burwitz, V. and Garmire, G.P. (2001) The X-ray spectrum of the Vela pulsar resolved with the Chandra X-ray observatory. Astrophys. J. Lett. 552, L129-L133.

49) Enoto, T., Nakazawa, K., Makishima, K., Rea, N., Hurley, K. and Shibata, S. (2010) Broadband study with Suzaku of the magnetar class. Astrophys. J. Lett. 722, L162-L167.

50) Enoto, T. et al. (2009) Suzaku observation of the new Soft Gamma Repeater SGR 0501+4516 in outburst. Astrophys. J. Lett. 693, L122-L126.

51) Enoto, T. et al. (2010) Suzaku discovery of a hard $\mathrm{X}$-ray tail in the persistent spectra from the magnetar 1E 1547.0-5408 during its 2009 activity. Publ. Astron. Soc. Jpn. 62, 475-485.

52) Enoto, T. et al. (2010) Wide-band Suzaku analysis of the persistent emission from SGR $0501+4516$ during the 2008 outburst. Astrophys. J. 715, 665670 .

53) Nakagawa, Y., Makishima, K. and Enoto, T. (2011) The Suzaku discovery of a hard power-law component in the spectra of short bursts from SGR 0501+4516. Publ. Astron. Soc. Jpn. 63, S813-S820.

54) Enoto, T., Nakagawa, Y.E., Sakamoto, T. and Makishima, K. (2012) Spectral comparison of weak short bursts to the persistent X-rays from the magnetar 1E 1547.0-5408 in its 2009 outburst. Mon. Not. R. Astron. Soc. 427, 2824-2840.

55) Iwahashi, T., Enoto, T., Yamada, S., Nishioka, H., Nakazawa, K., Tamagawa, T. and Makishima, K. (2013) Suzaku follow-up observation of the activated magnetar 1E 1547.0-5408. Publ. Astron. Soc. J. 65, id.52.

56) Nakagawa, Y., Yoshida, A., Yamaoka, K. and Shibazaki, N. (2009) Uniting the quiescent emission and burst spectra of magnetar candidates. Publ. Astron. Soc. Jpn. 61, 109-122.

57) Nakagawa, Y. et al. (2007) A comprehensive study of short bursts from SGR1806-20 and SGR1900+14 detected by HETE-2. Publ. Astron. Soc. Jpn. 59, 653-678.

58) Figer, D.F., Najarro, F., Geballe, T.R., Blum, R.D. and Kudritzki, R.P. (2005) Massive stars in the SGR 1806-20 cluster. Astrophys. J. Lett. 622, L49-L52.

59) Nakano, T. (2015) Suzaku studies of supernova remnants hosting non-rotation-powered neutron stars. Ph.D. Thesis, The University of Tokyo.

60) Nakano, T., Murakami, H., Makishima, K., Hiraga, J.S., Uchiyama, H., Kaneda, H. and Enoto, T. (2015) Suzaku studies of the supernova remnant CTB 109 hosting the magnetar 1E 2259+586. Publ. Astron. Soc. J. 67, id.9.

61) Rea, N. et al. (2010) A low-magnetic-field soft gamma repeater. Science 330, 944-947.

62) Rea, N., Viganó, D., Israel, G.L., Pons, J.A. and Torres, D.F. (2014) 3XMM J185246.6+ 003317: Another low magnetic field magnetar. Astrophys. J. Lett. 781, id.L17.

63) Takiwaki, T., Kotake, K. and Sato, K. (2009) Special relativistic simulations of magnetically dominated jets in collapsing massive stars. Astrophys. J. 691, $1360-1379$.

64) Kashiyama, K. and Ioka, K. (2011) Magnetar asteroseismology with long-term gravitational waves. Phys. Rev. D 83, id.081302.

65) Butikov, E. (2006) Inertial rotation of a rigid body. Eur. J. Phys. 27, 913-922.

66) Enoto, T., Makishima, K., Nakazawa, K., Kokubun, M., Kawaharada, M., Kotoku, J. and Shibazaki, N. (2011) Soft and hard X-ray emissions from the 
anomalous X-ray pulsar 4U $0142+61$ observed with Suzaku. Publ. Astron. Soc. Jpn. 63, 387-396.

67) Makishima, K., Enoto, T., Hiraga, J.S., Nakano, T., Nakazawa, K., Sakurai, S., Sasano, M. and Murakami, H. (2014) Possible evidence for free precession of a strongly magnetized neutron star in the magnetar $4 \mathrm{U} 0142+61$. Phys. Rev. Lett. 112 , id.171102.

68) Makishima, K., Enoto, T., Murakami, H., Furuta, Y., Nakano, T., Sasano, M. and Nakazawa, K. (2016) Evidence for a $36 \mathrm{ks}$ phase modulation in the hard X-ray pulses from the magnetar $1 \mathrm{E}$ 1547.0-5408. Publ. Astron. Soc. Jpn. 68 (in press).

69) Dall'Osso, S., Shore, S.N. and Stella, L. (2009) Early evolution of newly born magnetars with a strong toroidal field. Mon. Not. R. Astron. Soc. 398, 1869-1885.

70) Taylor, J.H. and Manchester, R.N. (1977) Recent observations of pulsars. Annu. Rev. Astron. Astrophys. 15, 19-44.

71) van den Heuvel, E.P.J. (1984) Models for the formation of binary and millisecond radio pulsars. J. Astrophys. Astron. 5, 209-233.

72) Itoh, N., Kotouda, T. and Hiraki, K. (1995) The nonexponential evolution of pulsar magnetic fields. III. Comparison with the new velocity measurement. Astrophys. J. 455, 244-251.

73) Enoto, T., Sasano, M., Yamada, S., Tamagawa, T.,
Makishima, K., Pottschmidt, K., Marcu, D., Corbet, R.H.D., Fuerst, F. and Wilms, J. (2014) Spectral and timing nature of the symbiotic X-ray binary $4 \mathrm{U} 1954+319$ : The slowest rotating neutron star in an X-ray binary systtem. Astrophys. J. 786, id.127.

74) Alpar, A., Pines, D., Anderson, P.W. and Shaham, J. (1984) Vortex creep and the internal temperature of neutron stars. I - General theory. Astrophys. J. 276, 325-334.

75) Rice, M.J. (1969) The hard-sphere Fermi gas and ferromagnetism in neutron stars. Phys. Lett. A 29, 637-638.

76) Manchester, R.N., Hobbs, G.B., Teoh, A. and Hobbs, M. (2005) The australia telescope national facility pulsar catalogue. Astron. J. 129, 1993-2006.

77) Lee, H.J., Canuto, V., Chiu, H.-Y. and Chiuderi, C. (1969) New state of ferromagnetism in degenerate electron gas and magnetic fields in collapsed bodies. Phys. Rev. Lett. 23, 390-393.

78) Onishi, N. (2015) Chiral asymmetry model for magnetars (private communication).

79) Ohnishi, A. and Yamamoto, N. (2014) Magnetars and the chiral plasma instabilities. astro-ph/ 1402.4760 .

(Received Oct. 1, 2015; accepted Feb. 29, 2016)

\section{Profile}

Kazuo Makishima, born in Tokyo in 1949, graduated from the University of Tokyo in 1972, and entered the research field of experimental astrophysics, mainly using scientific satellites to observe X-rays from various celestial objects. He became in 1978 Associate Professor at the Institute of Space and Astronautical Science, the University of Tokyo, and was promoted in 1986 to Associate Professor at the Department of Physics of the same university. After further promoted to Professor in 1995, he worked there till 2015. Meantime, he contributed, both in hardware developments and scientific observations, to all the 6 Japanese X-ray astronomy satellites, Hakucho (launched in 1979), Tenma (1983), Ginga (1987), ASCA (1993), Suzaku (2005), and Hitomi (just launched in 2016). Furthermore, he contributed to two solar satellites, Hinotori (1981)

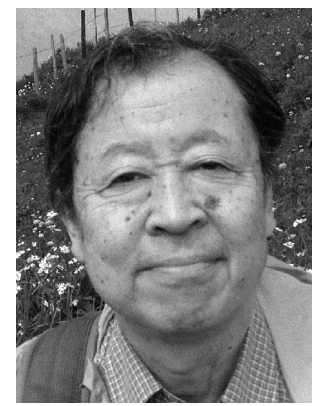
and Yohkoh (1991). From 2001 onwards, he was also jointly appointed as Chief Scientist (Group Director since 2009) at RIKEN, or The Institute of Physical and Chemical Research, where he supported the MAXI (Monitor of All-sky X-ray Image) instrument placed on the Japanese experimental module comprising the International Space Station. Using these space missions, he has so far achieved a number of new observational discoveries that allow us to better understand, e.g., magnetism of neutron stars, accretion processes onto black holes, and plasma-physics aspects of clusters of galaxies. At least for a decade since 1991, the Hard X-ray Telescope onboard Yohkoh, developed by him and his co-workers, held the record of the highest angular resolution, 5 arcseconds, in hard X-rays up to $100 \mathrm{keV}$. 